\title{
Ultrafast ultrasound imaging as an inverse problem: Matrix-free sparse image reconstruction
}

\author{
Adrien Besson, Student member, Dimitris Perdios, Student member, Florian Martinez, Zhouye Chen, Student \\ member, Rafael E. Carrillo, Member, Marcel Arditi, Senior member, Yves Wiaux, Jean-Philippe Thiran, Senior \\ member
}

\begin{abstract}
Conventional ultrasound (US) image reconstruction methods rely on delay-and-sum (DAS) beamforming, which is a relatively poor solution of the image reconstruction problem. An alternative to DAS consists in using iterative techniques which require both an accurate measurement model and a strong prior on the image under scrutiny. Towards this goal, much effort has been deployed in formulating models for US imaging which usually require a large amount of memory to store the matrix coefficients. We present two different techniques which take advantage of fast and matrix-free formulations derived for the measurement model and its adjoint, and rely on sparsity of US images in well-chosen models. Sparse regularization is used for enhanced image reconstruction. Compressed beamforming exploits the compressed sensing framework to restore high quality images from fewer raw-data than state-of-the-art approaches. Using simulated data and in vivo experimental acquisitions, we show that the proposed approach is three orders of magnitude faster than non-DAS state-of-the-art methods, with comparable or better image quality.
\end{abstract}

Index Terms-Ultrafast ultrasound imaging, compressed sensing, beamforming, sparse regularization

\section{INTRODUCTION}

Ultrasound (US) imaging is one of the most used medical imaging modalities enabling real-time, safe and low-cost procedures such as fetal, abdominal and cardiac imaging. Recently, a new paradigm, denoted as ultrafast US imaging, has enabled US imaging to reach more than thousands of frames per second, paving the way to a whole range of new applications such as tracking of induced and intrinsic shear waves in organs [1].

Pulse-echo US imaging uses an array of transducer elements to transmit short acoustical pulses through the body. The backscattered echo waveforms are then received by the same array and detected as so-called element raw-data. Retrieving an image of the medium inhomogeneities from the element raw-data poses an ill-posed inverse problem. Current real-time US imaging is generally based on the well-known delay-andsum (DAS) beamforming algorithm which can be seen as a back-projection solution of the inverse problem under several

A. Besson, D. Perdios, F. Martinez, M. Arditi and J.-Ph. Thiran are with the Signal Processing Laboratory 5 (LTS5), Ecole Polytechnique Fédérale de Lausanne, CH-1015, Lausanne, Switzerland.

R. E. Carrillo is with the Centre Suisse d'électronique et microtechnique (CSEM), CH-2002 Neuchâtel, Switzerland.

Z. Chen and Y. Wiaux are with the Institute of Sensors, Signals and Systems, Heriot-Watt University, EH14 4AS Edinburgh, United-Kingdom.

J.-Ph. Thiran is also with the Department of Radiology, University Hospital Center (CHUV) and University of Lausanne (UNIL), CH-1011, Lausanne, Switzerland assumptions [2]. While being suitable for real-time imaging, DAS suffers from a relatively low quality, in terms of signalto-noise ratio, and requires sampling the element raw-data at a rate few times higher than the Nyquist rate for sufficient accuracy in the delay calculations [3], [4].

An alternative to back-projection methods consists of sparse regularization (SR) techniques [5]. These methods are built upon forward models of the problem and introduce additional information on the signal under scrutiny in order to solve the ill-posed inverse problem, leading to a higher image quality than back-projection methods.

Medical imaging is well-suited to SR methods. Indeed, in many medical imaging modalities, the image reconstruction process amounts to solving a linear inverse problem. In magnetic resonance imaging (MRI), the image is reconstructed from k-space samples and the measurement model is an inverse Fourier transform [6]. In X-ray computed tomography $(\mathrm{CT})$, the sinogram is related to the measurements by the Beer-Lambert law which can be expressed as a linear inverse problem in the discrete domain [7]. Moreover, sparsity priors have been expressed for medical images. Lustig et al. [6] have exploited sparsity of MRI images in the wavelet domain and under the total-variation (TV) transform. In X-ray CT, sparsity priors under the TV-norm have been extensively used [8], [9].

In US imaging, several formulations of forward models have been recently investigated. David et al. [10], Wang et al. [11] and Besson et al. [12] have proposed time-domain formulations of the problem. In the Fourier domain, Zhang et al. [13] have suggested a formulation of the model derived by David et al. [10]. Besson et al. [14] have presented a forward model in the Fourier domain in which US propagation is seen as a projection on a non-uniform Fourier space. Schiffner and Schmitz [15] have proposed a time-frequency model in which each frequency of the transducer-element bandwidth is treated independently, enabling the model to deal with distortion effects. The main problem of these models resides in their computational complexity, usually translated in storage requirements of the corresponding matrix representation. The models proposed by David et al. [10] and Schiffner and Schmitz [15] require the storage of several hundreds of GB for matrix coefficients in 2D. Zhang et al. [13] have divided the image in stripes in order to make the problem tractable. This issue severely limits the appeal of iterative methods against classical approaches. Ozmen et al. [2] have suggested the use of matrix-free operators but did not provide any implementation detail and only applied their model to low 
frequencies.

SR methods have raised a growing interest recently in US imaging. They have been used for despeckling [16], [17] and deconvolution [18], [19] of radio-frequency images. They have been exploited to solve inverse scattering problems, e.g. using the constrast-source-inversion method [2]. They have also been used in the context of the compressed sensing (CS) framework. It has been explored in a pre-beamforming step, where the problem has been formulated on the element raw-data [20]. It has also been investigated in a post-beamforming step in order to reduce the amount of data to be stored [21], [22] as well as combined with a deconvolution framework for image enhancement [19]. In a similar direction, it has been used in the image reconstruction process, in order to reduce the amount of data necessary to retrieve a high quality image either by reducing the number of transducer elements [10], [12] or by sub-Nyquist rate sampling [4], and to improve the image quality compared to backprojection methods [13], [14], [15], [23], [24], [25], [26].

Three main contributions are proposed in this work. Firstly, parametric, fast and matrix-free formulations of the measurement model and its adjoint are described for both planewave (PW) and diverging-wave (DW) compounding. Secondly, a fully parallel implementation of these formulations is included in a sparse regularization framework, resulting in highquality imaging with near real-time capability and no memory footprint, paving the way to sparse regularization for 3D US imaging. Lastly, the proposed model is coupled with innovative compression strategies which outperform existing compressed beamforming (CB) schemes [10], [12].

The remainder of the paper is organized as follows. The matrix-free formulations of the measurement model and its adjoint are described in Section II. The proposed image reconstruction method is described in Section III and evaluated on simulated and in vivo data in Section IV. Results are reported in Sections V and VI and discussed in Section VII. Concluding remarks are given in Section VIII.

\section{PARAMETRIC MATRIX-FREE FORMULATIONS OF THE MEASUREMENT MODEL AND ITS ADJOINT}

\section{A. Formulation of the measurement model}

Ultrafast US imaging involves transmission of either steered PW (SPW) or DWs. In this case, it has been shown that one may express the image reconstruction as an inverse problem [10], [15]. More precisely, let us consider a pulseecho experiment, described on Figure 1, where the propagation medium $\Omega \in \mathbb{R}^{2} \backslash\{z \leq 0\}$ contains inhomogeneities as local fluctuations in acoustic velocity and/or density, defining a tissue reflectivity function (TRF) $\gamma(\boldsymbol{r})$ with $\boldsymbol{r}=[x, z]^{T} \in$ $\Omega$ [15], [27]. The medium is insonified with a 1D-array of $N_{e l}$ transducer elements, located at $\boldsymbol{r}_{t}=\left[x_{t}, 0\right]^{T}$, where $x_{t} \in$ $\Xi \subset \mathbb{R}$, and the echo signals detected by the same elements are

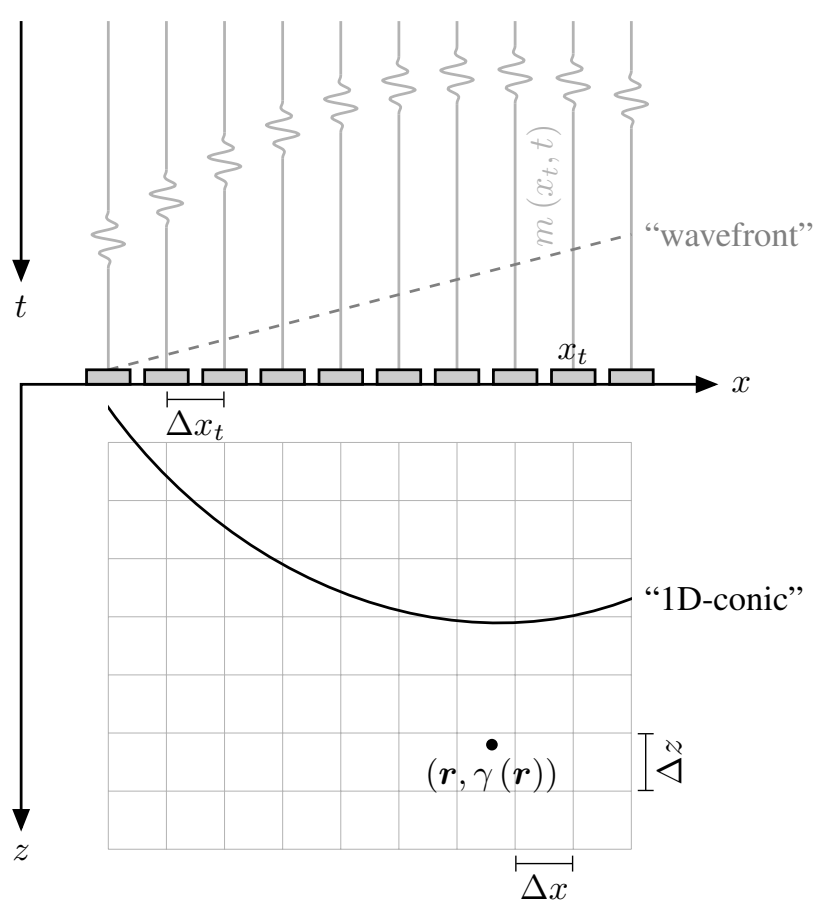

Fig. 1: Standard 2D-ultrafast ultrasound imaging configuration.

denoted as $m\left(x_{t}, t\right)$. Thus, the following relationship holds:

$$
\begin{aligned}
m\left(x_{t}, t\right)= & \int_{\boldsymbol{r} \in \Omega} o_{d}\left(\boldsymbol{r}, x_{t}\right) \gamma(\boldsymbol{r}) \\
& v_{p e}\left(t-t_{T x}(\boldsymbol{r})-t_{R x}\left(\boldsymbol{r}, x_{t}\right)\right) d \boldsymbol{r},
\end{aligned}
$$

which can be rewritten as:

$$
m\left(x_{t}, t\right)=\iint_{\tau \in \mathbb{R}, \boldsymbol{r} \in \Gamma\left(x_{t}, \tau\right)} \frac{o_{d}\left(\boldsymbol{r}, x_{t}\right) \gamma(\boldsymbol{r})}{\left|\nabla_{\boldsymbol{r}} g\right|} d \sigma(\boldsymbol{r}) v_{p e}(t-\tau) d \tau,
$$

where $v_{p e}(t)$ denotes the pulse-echo waveform, $o_{d}\left(\boldsymbol{r}, x_{t}\right)$ is defined as

$$
o_{d}\left(\boldsymbol{r}, x_{t}\right)=o\left(\boldsymbol{r}, x_{t}\right) /\left(2 \pi \sqrt{\left(x-x_{t}\right)^{2}+z^{2}}\right),
$$

with $o\left(\boldsymbol{r}, x_{t}\right)$ the element directivity according to Selfridge $e t$ al. [28], $g\left(\boldsymbol{r}, x_{t}, t\right)$ is defined as:

$$
g\left(\boldsymbol{r}, x_{t}, t\right)=t-t_{T x}(\boldsymbol{r})-t_{R x}\left(\boldsymbol{r}, x_{t}\right),
$$

with $t_{T x}(\boldsymbol{r})$ the propagation delay in transmit and $t_{R x}\left(\boldsymbol{r}, x_{t}\right)$ the propagation delay in receive defined as:

$$
t_{R x}\left(\boldsymbol{r}, x_{t}\right)=\sqrt{\left(x-x_{t}\right)^{2}+z^{2}} / c,
$$

$\nabla_{\boldsymbol{r}} g$ denotes the gradient of $g$ with respect to the variable $\boldsymbol{r}$ and $d \sigma(\boldsymbol{r})$ is the measure over the 1D-curve $\Gamma\left(x_{t}, t\right)$ defined as:

$$
\Gamma\left(x_{t}, t\right)=\left\{\boldsymbol{r} \in \Omega \mid g\left(\boldsymbol{r}, x_{t}, t\right)=0\right\}
$$

This model is a pulse-echo-spatial-impulse-response model firstly introduced by Tupholme [29] and Stepanishen [30], later used by Jensen and Svendsen [31]. 
Equation (2) can be written as follows:

$$
m\left(x_{t}, t\right)=\int_{\tau \in \mathbb{R}} h\left(x_{t}, \tau\right) v_{p e}(t-\tau) d \tau,
$$

where

$$
h\left(x_{t}, t\right)=\int_{\boldsymbol{r} \in \Gamma\left(x_{t}, t\right)} \frac{o_{d}\left(\boldsymbol{r}, x_{t}\right) \gamma(\boldsymbol{r})}{\left|\nabla_{\boldsymbol{r}} g\right|} d \sigma(\boldsymbol{r}) .
$$

Let us now introduce the parametrization $z=z\left(x, x_{t}, t\right)$ of the 1D-conic described by $\Gamma\left(x_{t}, t\right)$. The curvilinear integral defined in Equation (8) can then be recast as the following 1D integral:

$$
\begin{aligned}
h\left(x_{t}, t\right)=\int_{x \in \mathbb{R}} \frac{o_{d}\left(x, z\left(x, x_{t}, t\right), x_{t}\right)}{\left|\nabla_{\left(x, z\left(x, x_{t}, t\right)\right)} g\right|} \\
\gamma\left(x, z\left(x, x_{t}, t\right)\right)\left|J_{z}(x)\right| d x,
\end{aligned}
$$

where $\left|J_{z}(x)\right|=\sqrt{1+\left(\frac{d z}{d x}\right)^{2}}$ is the Jacobian associated with the parameterization of the curve.

The parametric formulations used in Equation (8) depend on the transmit scheme and are expressed in Appendix A for PW and DW imaging, respectively.

Let us introduce the lateral position of the $j$-th transducer element, given by $x_{t}^{j}=x_{t}^{0}+j \Delta x_{t}$ with $\Delta x_{t}$ the pitch and $x_{t}^{0}$ the position of the reference transducer element. Let us also consider that the pulse-echo experiment is achieved at time samples $t^{l}=t^{0}+l \Delta t$, for $l \in\left\{1, \ldots, N_{t}\right\}$, where $\Delta t=1 / f_{s}$, $f_{s}$ is the sampling frequency and $t^{0}$ the initial recording time sample. Let us also introduce the image grid defined by $x^{n}=$ $x^{0}+n \Delta x$ and $z^{q}=z^{0}+q \Delta z$ with $(n, q) \in\left\{1, \ldots, N_{x}\right\} \times$ $\left\{1, \ldots, N_{z}\right\}$.

It is demonstrated, in Appendix B, that, for each samples of the element-raw data, the following relationship holds:

$$
m\left(x_{t}^{j}, t^{l}\right)=\mathcal{H}\left(\boldsymbol{\gamma}, x_{t}^{j}, t^{l}\right)+\nu^{\prime}\left(x_{t}^{j}, t^{l}\right),
$$

where $\gamma \in \mathbb{R}^{N_{x} \times N_{z}}$ are the values of the TRF evaluated on the image grid defined above, $\mathcal{H}$ is the matrix-free measurement model and $\nu^{\prime}\left(x_{t}^{j}, t^{l}\right)$ is the noise which accounts for the model, interpolation and measurement errors.

The signal received by the $j$-th transducer element can thus be written as a vector $\boldsymbol{m}_{j} \in \mathbb{R}^{N_{t}}$ which contains the backscattered echoes from the medium, recorded at time samples defined above. By concatenating the signals sensed by all the transducer elements in a single vector, one may come up with a measurement vector $\boldsymbol{m} \in \mathbb{R}^{N_{e l} N_{t}}$.

\section{$B$. The adjoint of the proposed measurement model and its relationship with the delay-and-sum algorithm}

Image reconstruction methods, either analytical or iterative, require the computation of the adjoint of the operator defined in Equation (10) [5]. Indeed, back-projection methods are based on applying the adjoint operator of the measurement model. In regularization approaches, the adjoint operator is used at each iteration of the reconstruction algorithms.

In this section, we derive the adjoint of the measurement model proposed in Section II-A. First, it is demonstrated that the adjoint operator of the continuous forward model described in Equation (2) can be written as:

$$
\begin{aligned}
& \hat{\gamma}(\boldsymbol{r})=\int_{x_{t} \in \Xi} o_{d}\left(\boldsymbol{r}, x_{t}\right) \\
&\left(m\left(x_{t}\right) *_{t} u\right)\left(t_{T x}(\boldsymbol{r})+t_{R x}\left(\boldsymbol{r}, x_{t}\right)\right) d x_{t},
\end{aligned}
$$

where $u(t)=v_{p e}(-t)$ is the matched filter corresponding to $v_{p e}$. The proof of Equation (11) is given in Appendix C.

Thus the parameterization of the adjoint operator is straightforward since $t=t_{T x}(\boldsymbol{r})+t_{R x}\left(\boldsymbol{r}, x_{t}\right)$. A matrix-free formulation of the adjoint operator can therefore be derived, for each point of the image grid, which leads to the following relationship:

$$
\hat{\gamma}\left(x^{n}, z^{q}\right)=\mathcal{H}^{\star}\left(\boldsymbol{m}, x^{n}, z^{q}\right)+\xi^{\prime}\left(x^{n}, z^{q}\right),
$$

where $\mathcal{H}^{\star}$ is the adjoint operator described in Appendix B and $\xi^{\prime}\left(x^{n}, z^{q}\right)$ is the noise which accounts for the model, interpolation and measurement errors.

The DAS algorithm is the standard image reconstruction method employed in US imaging because of its simplicity and real-time capability. The DAS algorithm can be mathematically formulated as:

$$
\gamma^{D A S}(\boldsymbol{r})=\int_{x_{t} \in \Xi} a\left(\boldsymbol{r}, x_{t}\right) m\left(x_{t}, t_{T x}(\boldsymbol{r})+t_{R x}\left(\boldsymbol{r}, x_{t}\right)\right) d x_{t},
$$

where $a\left(\boldsymbol{r}, x_{t}\right)$ accounts for the apodization weights, and $\gamma^{D A S}(\boldsymbol{r})$ is the DAS estimate of the TRF. In the light of Equation (11), it is demonstrated that DAS achieves a backprojection solution of the inverse problem when the effect of the pulse-echo waveform is neglected ( $u$ considered as a Dirac) and when the apodization weights $a\left(\boldsymbol{r}, x_{t}\right)$ are set to be equal to $o_{d}\left(\boldsymbol{r}, x_{t}\right)$.

Such solution can therefore be straightforwardly defined in the context of the adjoint operator $\mathcal{H}^{\star}$ :

$$
\gamma^{D A S}\left(x^{n}, z^{q}\right)=\mathcal{H}^{D A S}\left(\boldsymbol{m}, x^{n}, z^{q}\right)+\xi^{D A S}\left(x^{n}, z^{q}\right),
$$

where $\mathcal{H}^{D A S}$ denotes the approximation of the operator $\mathcal{H}^{\star}$ and $\xi^{D A S}\left(x^{n}, z^{q}\right)$ is the corresponding noise, taking into account the assumptions of DAS.

The DAS solution, while suited to real-time imaging, is therefore a relatively poor approximation of the solution of Problem (2). Indeed, DAS beamforming does not address the problem of the noise $\boldsymbol{n}$, neglects pulse-shape, and requires a sampling rate higher than Nyquist sampling requirements because of the high-delay resolution required [3], [4]. Indeed, to apply the delay defined in Equation (13) digitally, received signals must be sampled on a sufficiently dense grid.

\section{The inverse problem of interest}

In the literature, two different groups of image reconstruction methods may be distinguished, since they aim at solving two different problems.

The first group of methods can be denoted as regularized beamforming methods. They do not take into account the pulse-echo waveform and retrieve a low-resolution estimate 
of the TRF $\gamma$, usually denoted as the radio-frequency (RF) image $\gamma^{R F}$ [11], [12], [23], [24], [32], [33]. One reason for this choice resides in the fact that $\gamma^{R F}$ preserves speckle information (due to the low-resolution), which is of interest in many US applications.

The second group of methods aims at performing inverse scattering, i.e. at retrieving the high-resolution TRF $\gamma$ from the element-raw data [2], [10], [15]. This problem is far more complex, highly ill-posed, and usually requires more sophisticated models than the ones used for regularized beamforming methods.

While the model described in Section II can be used for inverse scattering problems, we focus, in this work, on regularized beamforming, which means that we neglect the effect of the pulse-echo waveform $v_{p e}$ in the model. This results in approximating the pulse-echo waveform as a Dirac delta function and Equation (2) is greatly simplified as:

$$
m\left(x_{t}, t\right)=\int_{\boldsymbol{r} \in \Gamma\left(x_{t}, t\right)} \frac{o_{d}\left(\boldsymbol{r}, x_{t}\right) \gamma(\boldsymbol{r})}{\left|\nabla_{\boldsymbol{r}} g\right|} d \sigma(\boldsymbol{r}) .
$$

Moreover, it can be noticed that Equation (10) can be expressed using a matrix formalism, leading to a more standard formulation of the inverse problem as:

$$
\boldsymbol{m}=\mathrm{H}^{R F} \gamma^{R F}+\boldsymbol{\nu}^{R F},
$$

where $\mathrm{H}^{R F} \in \mathbb{R}^{N_{e l} N_{t} \times N_{x} N_{z}}$ and $\boldsymbol{\nu}^{R F} \in \mathbb{R}^{N_{e l} N_{t}}$ are the operator and the noise evaluated at each sample of the elementraw data grid.

In the light of Problem (16), the DAS estimate described in Section II-B can be seen as a low-quality estimate of $\gamma^{R F}$.

\section{SPARSITY-DRIVEN IMAGE RECONSTRUCTION METHODS}

\section{A. A quick tour on sparse regularization and compressed sensing}

1) Sparse regularization: Regularization techniques introduce additional prior information to solve an ill-posed linear inverse problem of the form $\boldsymbol{y}=\boldsymbol{\Phi}+\boldsymbol{n}$, where $\boldsymbol{y} \in \mathbb{C}^{M}$ are the measurements, $s \in \mathbb{C}^{N}$ is the image under scrutiny, $\Phi \in \mathbb{C}^{M \times N}$ is the measurement model and $\boldsymbol{n} \in \mathbb{C}^{M}$ is the observation noise. The problem associated with regularization techniques is of the following form:

$$
\min _{\boldsymbol{s}} \mathcal{F}(\boldsymbol{\Phi} \boldsymbol{s}, \boldsymbol{y})+\beta \mathcal{R}(\boldsymbol{s}),
$$

where $\mathcal{F}$ is a distance function between $\Phi \boldsymbol{s}$ and $\boldsymbol{y}, \mathcal{R}$ is a nonnegative functional, which accounts for the prior information on $s$, and $\beta$ denotes the regularization parameter. One wellknown technique is Tikhonov regularization in which $\mathcal{R}$ is the $\ell_{2}$-norm. It has been studied by Szasz et al. [33] in the case of US image reconstruction.

SR techniques offer an alternative to Tikhonov regularization and exploit a sparsity prior of the signals of interest under a given model $\Psi$, usually expressed in terms of the $\ell_{1}$-norm. The following problem is thus expressed:

$$
\min _{\boldsymbol{s}}\|\boldsymbol{\Phi}-\boldsymbol{y}\|_{2}^{2}+\lambda\left\|\Psi^{\dagger} \boldsymbol{s}\right\|_{1},
$$

where $\Psi^{\dagger}$ accounts for the adjoint operator of $\psi$ and $\lambda$ is the regularization parameter. Problem (18) can also be written as:

$$
\min _{\boldsymbol{s}}\left\|\Psi^{\dagger} \boldsymbol{s}\right\|_{1} \text { subject to }\|\boldsymbol{y}-\Phi \boldsymbol{s}\|_{2} \leq \epsilon,
$$

where $\epsilon$ is a upper bound of the $\ell_{2}$-norm of the noise. Problems (18) and (19) can be solved using convex-optimization algorithms such as primal-dual-forward-backward (PDFB) [34], used in the proposed work and described in Appendix D.

2) Compressed sensing: The now famous theory of CS introduces a signal acquisition framework that goes beyond the traditional Nyquist sampling paradigm [35], [36], [37]. CS demonstrates that sparse or compressible signals can be acquired using a small number of linear measurements and recovered by solving a non-linear optimization problem [35], [36], [37].

Formally, the signal $s$ is acquired through the linear measurement model $\Phi \in \mathbb{C}^{M \times N}$ and $\boldsymbol{y}=\boldsymbol{\phi}+\boldsymbol{n}$ as defined in Section III-A1. When $M<N$, recovering $s$ from $\boldsymbol{y}$ amounts to solving an ill-posed inverse problem and CS demonstrates that $s$ can be recovered exactly from $\boldsymbol{y}$, with high probability, by solving Problem (19).

If we consider a matrix $\mathrm{A}=\left[\boldsymbol{a}_{1}, \ldots, \boldsymbol{a}_{L}\right] \in \mathbb{C}^{M \times L}$, its mutual coherence is measured as:

$$
\mu(\mathrm{A})=\max _{k, j, k \neq j} \frac{\left|<\boldsymbol{a}_{k}, \boldsymbol{a}_{j}\right\rangle \mid}{\left\|\boldsymbol{a}_{k}\right\|_{2} \cdot\left\|\boldsymbol{a}_{j}\right\|_{2}},
$$

where $\langle\cdot, \cdot\rangle$ accounts for the inner-product in $\mathbb{C}^{M}$.

Theorem 3.1 from [35] tells us that the perfect recovery condition of $s$ relies on a trade-off between the coherence of A and the sparsity of $\psi^{\dagger} s$. It can be shown that $\mu(\mathrm{A})$ lies between the Welch bound [38] and 1. The Welch bound gives a higher bound on the sparsity of the solution [35]. Putting everything together, to maximize the benefits of Theorem 3.1 from [35], one should be able to:

- build a measurement model $\Phi$ in such a way that $\mu(\Phi \Psi)$ is as close as possible to the Welch bound;

- build a sparsity model $\Psi$ in such a way that $\alpha=\Psi^{\dagger} s$ is as sparse as possible.

\section{B. Sparse regularization for enhanced ultrasound image re- construction}

SR for enhanced US image reconstruction proposes an alternative to DAS for solving Problem (16) by exploiting the SR framework described in Section III-A1. Indeed, Problem (16) is an ill-posed linear inverse problem, thus suited to the SR framework. The underlying idea consists of the introduction of a sparsity prior on RF images in a well-chosen model, described hereafter, in order to recover a high quality image by solving Problem (19).

The sparsifying model $\Psi$, denoted as sparsity averaging (SA) is composed of a concatenation of 8 Daubechies wavelet transforms with different mother wavelets ranging from Daubechies 1 (db1) to Daubechies 8 (db8) [39]. The operator is written as:

$$
\Psi=\frac{1}{\sqrt{q}}\left[\Psi_{1}, \ldots, \Psi_{q}\right]
$$


where $q$ is equal to 8 and $\Psi_{i}$ denotes the $i$-th Daubechies wavelet transform. The choice of the SA model is justified by its superior results on US images [14], compared to simpler wavelet-based models.

\section{Compressed beamforming}

$\mathrm{CB}$ aims at reducing the amount of data needed to recover high-quality US images. It is built upon the CS framework described in Section III-A2.

To account for the undersampling process, supposed to be linear, we introduce a matrix $\mathrm{D} \in \mathbb{R}^{M \times N_{e l} N_{t}}$, with $M \leq$ $N_{e l} N_{t}$, such that $\boldsymbol{m}_{d}=\mathrm{D} \boldsymbol{m}$ and $\mathrm{H}_{d}=\mathrm{DH}^{R F}$. The inverse problem can be naturally expressed as:

$$
\boldsymbol{m}_{d}=\mathrm{H}_{d} \gamma^{R F}+\nu_{d},
$$

where $\boldsymbol{\nu}_{d} \in \mathbb{R}^{M}$ accounts for the noise.

CB uses SR approaches, described in Section III-A, to solve Problem (22). The sparsity prior is expressed in the SA model and $\mathrm{CB}$ exploits the requirements of Theorem 3.1 from [35] for the design of the undersampling operator $D$.

The perfect recovery condition of $\mathrm{CB}$ relies on the incoherence between the measurement model $\mathrm{H}_{d}$ and the sparsifying model $\Psi$. In the case of US imaging, the measurement model $\mathrm{H}$ is imposed by the problem and the undersampling operator $D$ should be defined in such a way that the coherence between $\mathrm{H}_{d}$ and $\Psi$ is minimized.

1) Selection of transducer elements: In our previous work [12], we have proposed several designs for the undersampling operator which selects a subset of transducer elements, either uniformly- or randomly-spaced. In this case, the operator D selects the transducer elements of interest. It has been shown that high quality reconstructions may be achieved with approximately $40 \%$ to $50 \%$ of the initial data, for PW and DW imaging [32]. However, the corresponding measurement model $\mathrm{H}_{d}$ suffers from a high coherence [10], [12].

2) Mixing of the raw data: Taking into account the increase of the coherence induced by these simple undersampling schemes, one may think about designing a strategy such that the mutual coherence of the measurement operator is optimized. The underlying idea is to spread the information as much as possible between the element raw-data space and the desired-image space, hence lowering the coherence, similarly to sparse MRI acquisition [6]. Put formally, we introduce a linear operator $\mathrm{W} \in \mathbb{R}^{N_{e l} N_{t} \times N_{e l} N_{t}}$ such that $\mathrm{D}=\mathrm{PW}$, where $\mathrm{P} \in \mathbb{R}^{M \times N_{e l} N_{t}}$ represents an undersampling operator.

The relationship between the desired-image space and the element raw-data space is described by projections onto 1Dconics. It can be deduced that only few samples of the desiredimage space are contributing to every samples of the element raw-data space, leading to a highly coherent measurement model.

Nevertheless, it is interesting to note that each point of the element raw-data space generates a different conic in the desired-image space. Since two non-identical conics may intersect on four points at most, the information contained in different samples of the element raw-data space are complementary. Taking into account such an observation, our idea consists in mixing the information contained in various points of the element raw-data space in order to increase the amount of information carried by each measurement, which may lead to a lower coherence of the matrix.

Formally, the matrix $\mathrm{W}$ is designed as a random matrix, i.e. with i.i.d. entries drawn from a probability distribution. If we denote by $m\left(x_{t}^{j}, t^{l}\right)$ the element raw-data received at sample $t^{l}$ by the transducer element positioned at $x_{t}^{j}$ and by $m_{W}\left(x_{t}^{k}, t^{u}\right)$ the mixed element raw-data samples at sample $t^{u}$ on the synthetic mixed channel $x_{t}^{k}$, the following equation holds:

$m_{W}\left(x_{t}^{k}, t^{u}\right)=\sum_{j=1}^{N_{e l}} \sum_{l=1}^{N_{t}} w_{j l k u} m\left(x_{t}^{j}, t^{l}\right), \forall k \in\left\{1, \ldots, N_{e l}\right\}$.

where $w_{j l k u}$ are drawn from a probability distribution. It can be observed that each mixed element raw-data $m_{W}\left(x_{t}^{k}, t^{u}\right)$ is related to $\gamma^{R F}\left(x^{n}, z^{q}\right)$ by an integration on $\Gamma_{W}\left(x_{t}^{k}, t^{u}\right)=$ $\bigcup_{l=1}^{N_{t}} \bigcup_{j=1}^{N_{e l}} \Gamma\left(x_{t}^{j}, t^{l}\right)$, where $\Gamma\left(x_{t}^{j}, t^{l}\right)$ is a 1D-conic defined in Section II-A. Since each 1D-conic carries complementary information about the desired-image space, it can be stated that $\Gamma\left(x_{t}^{j}, t^{l}\right) \subset \Gamma_{W}\left(x_{t}^{k}, t^{u}\right)$.

Finally, one can vectorize Equation (23) in order to come up with the following equation:

$$
\boldsymbol{m}_{d}=\mathrm{PW} \boldsymbol{m}
$$

The main drawback of this strategy resides in the design of the linear operator W. As an example, if we consider element raw-data acquired over 1000 depth samples, with 128 transducer elements, and that the grid of the desired image is the same as the grid of the element raw-data, then $\mathrm{W}$ requires $131 \mathrm{~GB}$ of memory to store the matrix coefficients, in double precision. In order to overcome this drawback, two strategies, denoted as 'Channel mixing' (CMIX) and 'Channel and time mixing' (CTMIX) are proposed.

CMIX consists of a random summation of the signals coming from the different transducers elements at a given time instant in order to create a mixed output. In this case, Equation (23) becomes:

$$
m_{W}\left(x_{t}^{k}, t^{u}\right)=\sum_{j=1}^{N_{e l}} w_{j k u} m\left(x_{t}^{j}, t^{u}\right), \forall k \in\left\{1, \ldots, N_{e l}\right\}
$$

The matrix $\mathrm{W}$ only contains $N_{e l}$ values per row which drastically reduces its complexity. In addition, such a mixing could be achievable in hardware, providing a probe which integrates a system able to perform CMIX in a pre-beamforming step. However, it is clear that the mixing and resulting coherence reduction effects of CMIX are rather limited compared to a fully random mixing.

CTMIX extends the principle of CMIX by considering mixing across both transducer elements and $D_{t}$ time samples 
to limit the complexity. Put formally, the following relationship holds:

$m_{W}\left(x_{t}^{k}, t^{u}\right)=\sum_{j=1}^{N_{e l}} \sum_{l=1}^{D_{t}} w_{j l k u} m\left(x_{t}^{j}, t^{l}\right), \forall k \in\left\{1, \ldots, N_{e l}\right\}$,

in which the samples $t^{l}$ are chosen randomly in the entire range of time. When $D_{t}=N_{t}$, CTMIX is equivalent to the case where a fully random matrix is used. When $D_{t}=1$ and $t^{l}=t^{u}$, CTMIX is equivalent to CMIX.

\section{EXPERIMENTS}

\section{A. Experimental setup}

1) Plane wave imaging: All the measurements (except the ones from the PICMUS dataset) are achieved with a standard linear-probe (designed to work at $7.8 \mathrm{MHz}$ ) whose settings are given in Table I. For each experiment, a sequence of 15 SPWs (5 MHz, 1-cycle, tri-state waveforms) is transmitted with steering angles uniformly distributed between $-7.5^{\circ}$ and $7.5^{\circ}$. No apodization is used on transmit.

TABLE I: Characteristics of the ATL L12-5 $50 \mathrm{~mm}$ probe used for PW imaging.

\begin{tabular}{|ll|}
\hline Parameter & Value \\
\hline Number of elements & 128 \\
Center frequency & $5 \mathrm{MHz}$ \\
Wavelength & $0.31 \mathrm{~mm}$ \\
Sampling frequency & $31.2 \mathrm{MHz}$ \\
Pitch & $0.195 \mathrm{~mm}$ \\
Kerf & $0.05 \mathrm{~mm}$ \\
\hline
\end{tabular}

- Numerical simulations: The system described above is simulated using Field II software [31]. Two phantoms are insonified:

- Point-reflector phantom: It consists of bright reflectors laterally positioned at $5 \mathrm{~mm}$ and spaced in depth by $10 \mathrm{~mm}$. At depths of $10 \mathrm{~mm}$ and $30 \mathrm{~mm}$, bright reflectors are distributed laterally with a step of $5 \mathrm{~mm}$.

- Anechoic-inclusion phantom: It consists of an anechoic inclusion of $8 \mathrm{~mm}$ diameter, centered at a depth of $40 \mathrm{~mm}$, embedded in a medium with a high density of scatterers with random positions and amplitudes (20 scatterers per resolution cell).

- PICMUS dataset: The methods are also evaluated on the standardized PICMUS dataset [40]. More precisely, the simulated dataset as well as the in vivo carotids are used. The corresponding settings are available on the PICMUS website $^{1}$.

- In vivo experiments: Two in vivo carotid images were acquired using a Verasonics US scanner (Redmond, WA, USA) with the ATL probe whose settings are given in Table I.

\footnotetext{
${ }^{1}$ https://www.creatis.insa-lyon.fr/Challenge/IEEE_IUS_2016/home
}

2) Diverging wave imaging: All the measurements are performed with a simulated phased-array probe whose settings are given in Table II. For each experiment, a sequence of 15 DWs (2.5 MHz, 1-cycle, tri-state waveforms) is transmitted with virtual point sources located at $z_{n}$ equal to $-2.9 \mathrm{~mm}$ and uniformly distributed at $x_{n}$ between $-5.9 \mathrm{~mm}$ and $5.9 \mathrm{~mm}$. No apodization is used on transmit.

TABLE II: Characteristics of the probe used for DW imaging.

\begin{tabular}{|ll|}
\hline Parameter & Value \\
\hline Number of elements & 64 \\
Center frequency & $2.5 \mathrm{MHz}$ \\
Wavelength & $0.62 \mathrm{~mm}$ \\
Sampling frequency & $15.6 \mathrm{MHz}$ \\
Pitch & $0.32 \mathrm{~mm}$ \\
Kerf & $0.05 \mathrm{~mm}$ \\
\hline
\end{tabular}

- Numerical simulations: We exploit the numerical simulation of the following phantoms:

- Point-reflector phantom: It consists of bright reflectors centered in the field and spaced in depth every $20 \mathrm{~mm}$. At $50 \mathrm{~mm}$ depth, bright reflectors are laterally distributed with a step of $20 \mathrm{~mm}$.

- Anechoic-inclusion phantom: The anechoic-inclusion phantom is the same as for the PW experiment.

\section{B. Image recontruction methods}

The proposed image reconstruction methods are evaluated against classical DAS algorithm as well as against best stateof-the-art image reconstruction algorithms on the PICMUS dataset, based on the log-compressed B-mode image. The envelope image is extracted from the US RF-image through the Hilbert transform, log-compressed over a range of $40 \mathrm{~dB}$ and finally converted to 8-bit gray scale to get the B-mode image.

The DAS algorithm used in this study is the one described by Montaldo et al. [41] for PW imaging and by Papadacci et al. [42] for DW imaging. It is used with a linear interpolation for delay calculations and with a factor correcting for the obliquity. For the iterative approaches, the measurement model and the adjoint described in Section II are used with a linear interpolation. The hyper-parameters of the optimization algorithm are empirically tuned depending on the reconstruction method. The stopping criterion is set to be a maximum number of iterations.

\section{Computation time}

The proposed image reconstruction methods are implemented on a NVIDIA Titan X GPU card for evaluation in terms of computation time. The timings are calculated as an average computation time over 500 draws of each reconstruction method. It has to be noted that the proposed implementation is not optimized and a substantial gain may be achieved by working on simple acceleration strategies.

\section{RESUlTS: SPARSE REGULARIZATION FOR ENHANCED IMAGE RECONSTRUCTION}

As explained in Section III-B, SR is a powerful framework to solve ill-posed inverse problems. In this section, we expose 
the results obtained with SR on the simulated point-reflector and anechoic-inclusion phantoms (described in Section IV-A), for both PW and DW imaging. We also present results on the in vivo carotid images obtained with $\mathrm{PW}$ imaging. We finally compare SR to the best state-of-the-art mage reconstruction algorithms on the PICMUS dataset.

\section{A. Contrast study on the anechoic-inclusion phantom}

In order to evaluate image contrast, the anechoic-inclusion phantom is used. The proposed method is coupled with a sparsity prior in the SA model described in Section III-B. The maximum number of iterations is set to 50 . The $\mathrm{dB}$ contrastto-noise ratio (CNR) [43] is calculated on the normalized envelope image, i.e. on the envelope image divided by its maximum value, using the following formula:

$$
\mathrm{CNR}=20 \log _{10} \frac{\left|\mu_{t}-\mu_{b}\right|}{\sqrt{\frac{\sigma_{t}^{2}+\sigma_{b}^{2}}{2}}},
$$

where $\left(\mu_{t}, \mu_{b}\right)$ and $\left(\sigma_{t}^{2}, \sigma_{b}^{2}\right)$ are the means and the variances of the target inclusion and the background, respectively.

Figures $2 \mathrm{a}$ and $2 \mathrm{~b}$ display the CNR values for PW and DW imaging, respectively, with 1 insonification for the proposed approach and 1 to 15 insonifications for DAS. It can be noticed that, with only 1 insonification, the proposed method leads to a contrast similar to DAS with more than 9 insonifications, and considerably better than DAS for 1 to 9 insonifications.

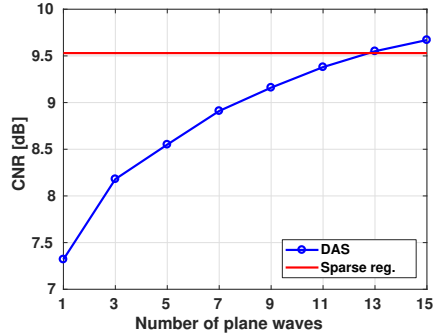

(a)

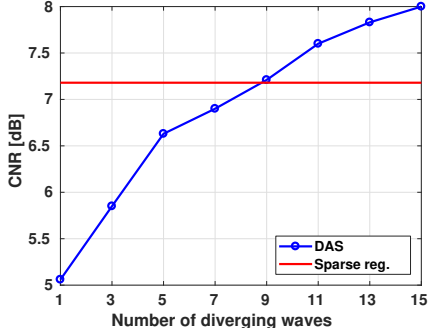

(b)
Fig. 2: CNR (in dB) calculated on the simulated anechoicinclusion phantom as a function of the number of insonifications for (a) PW imaging and (b) DW imaging. The solid-red line corresponds to the CNR of the proposed approach with 1 insonification and the dotted-blue line is the CNR obtained with DAS for 1 to 15 insonifications.

Figures $3 \mathrm{a}$ and $3 \mathrm{~b}$ display the B-mode images of the anechoic-inclusion phantom reconstructed with DAS (11 PW insonifications) and using the proposed approach (1 PW insonification), respectively. It can be noticed that the quality is comparable between the two images. The noise inside the anechoic area, due to the side lobes of the PSF, has been suppressed by the regularization. It can also be noticed that the speckle density is slightly decreased with the proposed approach, resulting in a darkening of the image in the deepest part of the Figure $3 \mathrm{~b}$. This effect is due to the fact that part of the speckle is not well preserved by the SA model, thus considered as noise and suppressed during the image reconstruction process [14].

\section{B. Reconstruction of the point-reflector phantom}

The point-reflector phantom is used to assess the quality of the proposed approach (100 iterations) in the particular case where only few sparse sources are present. One typical application is harmonic imaging of microbubbles in lowconcentrations where the individual responses of the sparse microbubbles are visible. The quality is evaluated on the resolution, calculated as the full width at half maximum (FWHM) of the point spread function.

For PW imaging, the proposed approach leads to lateral and axial resolutions of $0.10 \mathrm{~mm}$ for every points with 1 PW insonification, while the DAS algorithm gives lateral and axial resolutions of $0.20 \mathrm{~mm}$ for both points, constant for compounding experiments with 1 to $11 \mathrm{PW}$ insonifications. The increase is more pronounced in DW imaging where the proposed approach leads to lateral resolutions of $0.50 \mathrm{~mm}$ and $0.90 \mathrm{~mm}$ and axial resolutions of $0.50 \mathrm{~mm}$ for the points located at $30 \mathrm{~mm}$ and $50 \mathrm{~mm}$, while the DAS algorithm with 11 DW insonifications exhibits lateral resolutions of $1.40 \mathrm{~mm}$ and $2.80 \mathrm{~mm}$ and axial resolutions of $0.50 \mathrm{~mm}$.

The increase in resolution is visible when comparing Figures $3 \mathrm{c}$ and $3 \mathrm{~d}$, which display the B-mode images of the pointreflector phantom reconstructed with DAS (11 DW insonifications) and with the proposed approach (1 DW insonification), respectively.

\section{Reconstruction of the in vivo carotids}

The proposed method is evaluated on the in vivo carotid images. In this case, the SR is coupled with the SA model, the number of iterations is set to 50. Due to the lack of a ground truth image, the evaluation of the image quality is limited to visual assessment.

Figure 4 displays B-mode images for two different carotid acquisitions, reconstructed with the proposed method with 1 PW insonification and using DAS with 9 PW insonifications. It can be observed, by comparing Figure $4 \mathrm{a}$ with $4 \mathrm{~b}$ and Figure $4 \mathrm{c}$ with $4 \mathrm{~d}$ that the proposed method with $1 \mathrm{PW}$ leads to imaging quality similar to DAS with 9 PWs insonifications. Both the anechoic artery and the tissue area are well preserved with the proposed approach.

This illustrates the great potential of SR for reducing the number of insonifications required to reach a given image quality.

\section{Reconstruction of the PICMUS dataset}

In the above sections, the proposed approach has been compared to DAS. It is now well-known that DAS is not the highest quality beamforming and it could be interesting to compare the proposed approach to the best image reconstruction algorithms.

The proposed method is thus used to reconstruct simulated images from the standardized PICMUS dataset [40] and compared against two minimum-variance beamforming approaches [44], [45] and two sparse-based approaches [23], [24]. The results, displayed in Table III, show that the proposed method is competitive against the best state-of-the-art 


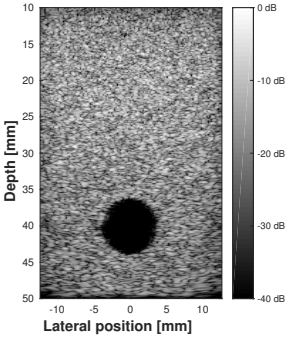

(a)

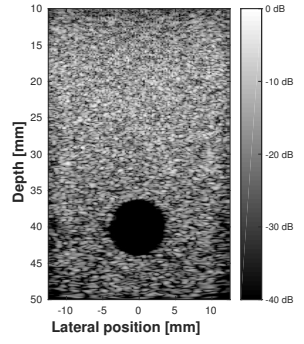

(b)

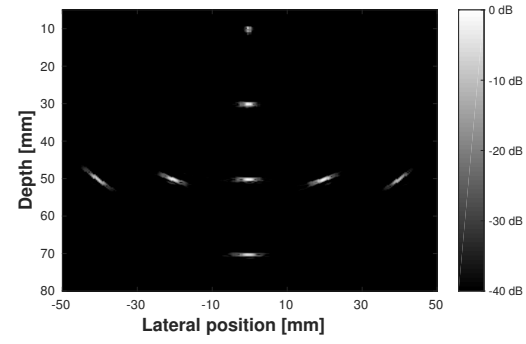

(c)

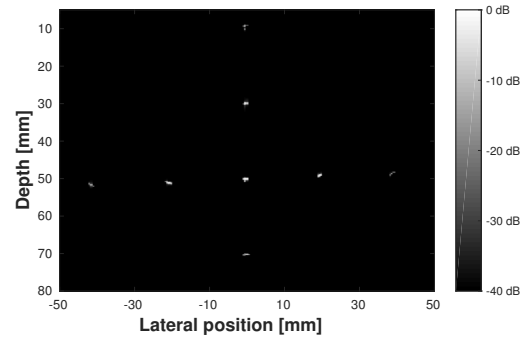

(d)

Fig. 3: B-mode images of the anechoic-inclusion phantom reconstructed with (a) DAS for 11 PW insonifications and (b) the proposed approach for $1 \mathrm{PW}$ insonification, and of the point-reflector phantom reconstructed with (c) DAS for 11 DW insonifications and (d) the proposed approach for $1 \mathrm{DW}$ insonification.

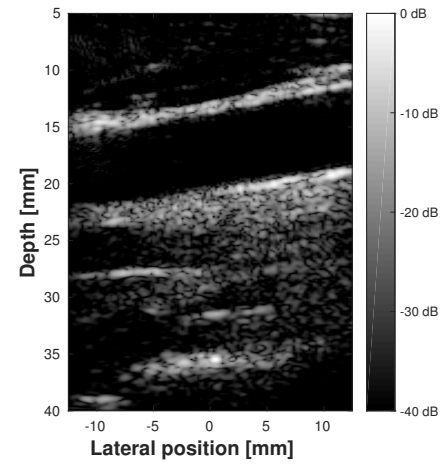

(a)

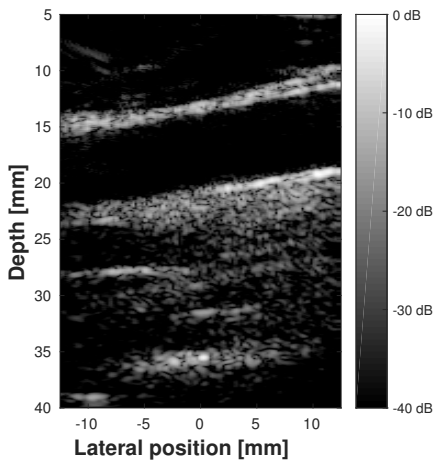

(b)

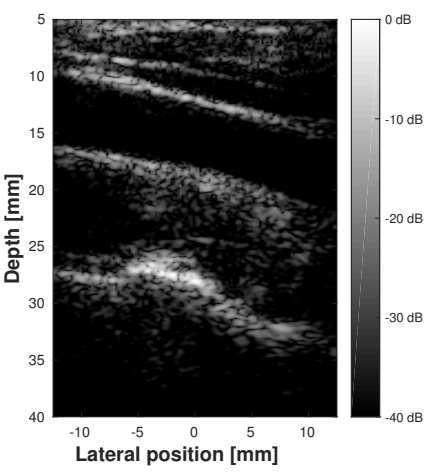

(c)

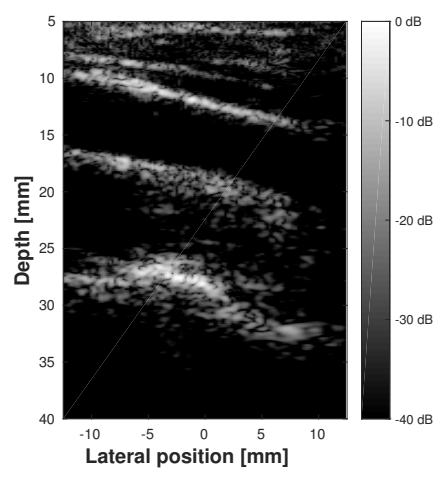

(d)

Fig. 4: B-mode images of two in vivo carotids reconstructed with DAS for 9 PW insonifications ((a) and (c)) and with the proposed approach for $1 \mathrm{PW}$ insonification ((b) and (d)).

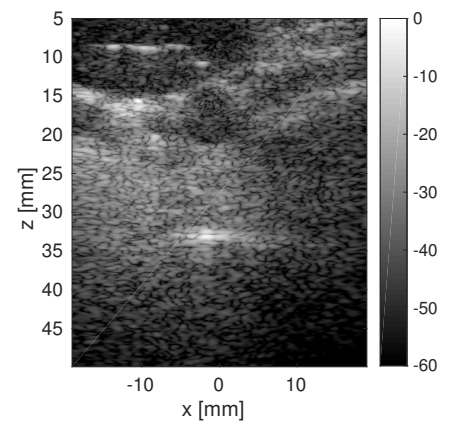

(a)

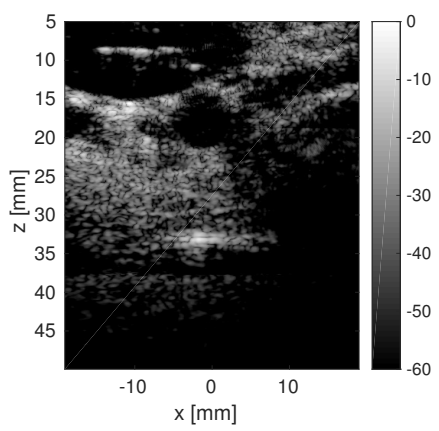

(b)

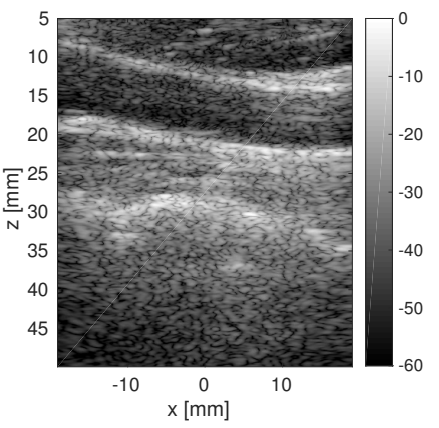

(c)

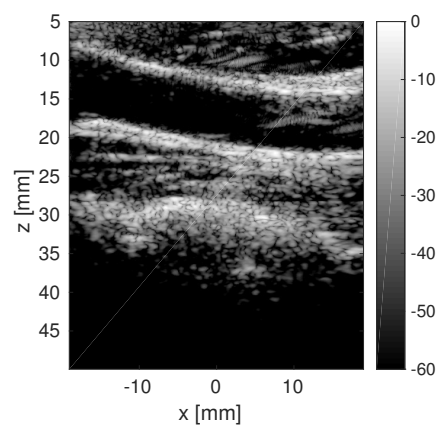

(d)

Fig. 5: B-mode images of the in vivo carotids of the PICMUS dataset for 1 PW insonification reconstructed with DAS ((a) and (c)) and with the proposed method ((b) and (d)).

approaches in terms of contrast. Regarding the resolution, the method outperforms the other approaches.

The proposed method is lastly used to reconstruct in vivo carotid images of the PICMUS dataset. The B-mode images, displayed on Figures $5 \mathrm{~b}$ and $5 \mathrm{~d}$ show a significant improvement compared to DAS, whose corresponding B-mode images are displayed on Figures $5 \mathrm{a}$ and $5 \mathrm{c}$, as already demonstrated in Section VI-D. A visual comparison of Figures $5 \mathrm{~b}$ and $5 \mathrm{~d}$ against images obtained with best state-of-the-art methods, that one can find on the PICMUS website ${ }^{2}$, shows that the

${ }^{2}$ https://www.creatis.insa-lyon.fr/Challenge/IEEE_IUS_2016/home proposed method outperforms most of them in terms of visual quality (in both contrast and resolution).

\section{E. Computation times for sparse regularization}

The computation times for the proposed sparse regularization method (50 iterations of PDFB) are $119 \mathrm{~ms}, 164 \mathrm{~ms}$ and $163 \mathrm{~ms}$ for the cyst phantom, the carotid displayed on Figure $4 \mathrm{~b}$ and the carotid displayed on Figure $4 \mathrm{~d}$ respectively. In the case of DW imaging, the point-reflector phantom displayed on Figure $3 \mathrm{~d}$ is reconstructed in $319 \mathrm{~ms}$.

These timings are two to three orders of magnitude faster than state-of-the-art methods. Indeed, in their latest work, 
TABLE III: Comparison of the proposed algorithm against state-of-the-art methods for sparse reconstruction on the simulated PICMUS dataset.

\begin{tabular}{|c|ccc|}
\hline \multirow{2}{*}{ Method } & Contrast ph. & \multicolumn{2}{c|}{ Resolution ph. } \\
& CNR [dB] & Lat. res. [mm] & Ax. res. [mm] \\
\hline DAS & 9.96 & 0.57 & 0.40 \\
Besson et al. [23] & 15.81 & 0.15 & 0.17 \\
Deylami et al. [45] & $\mathbf{1 7 . 1 9}$ & $\mathbf{0 . 0 8}$ & 0.24 \\
Szasz et al. [24] & 15.52 & 0.14 & $\mathbf{0 . 1 1}$ \\
Varray et al. [44] & 12.65 & 0.38 & 0.14 \\
Proposed method & 15.71 & $\mathbf{0 . 0 8}$ & $\mathbf{0 . 1 1}$ \\
\hline
\end{tabular}

David et al. [46] reported computation times between 3 and 5 min. Szasz et al. [33] reported reconstruction times between $10 \mathrm{~s}$ and $1 \mathrm{~min}$. The relative timings of the main operations

TABLE IV: Relative timings of the main operations involved in the proposed method for the cyst phantom for PW imaging and the point-reflector phantom for DW imaging.

\begin{tabular}{|ccc|}
\hline Operation & Plane wave (cyst.) & Diverging wave (point ref.) \\
$\mathrm{H} \boldsymbol{\gamma}$ & $22.1 \%$ & $32.9 \%$ \\
$\mathrm{H}^{\star} \boldsymbol{m}$ & $22.5 \%$ & $11.7 \%$ \\
$\boldsymbol{\Psi}_{\boldsymbol{\gamma}}$ & $24.9 \%$ & $25.5 \%$ \\
$\boldsymbol{\Psi}^{\star} \boldsymbol{\alpha}$ & $24.0 \%$ & $24.1 \%$ \\
Others & $6.5 \%$ & $5.8 \%$ \\
\hline
\end{tabular}

involved in the image reconstruction process are reported in Table IV. It can be observed that the timings are balanced between the different operations. It can also be noticed that the computation times of the operations $\mathrm{H} \gamma$ and $\mathrm{H}^{\star} \boldsymbol{m}$ highly depend on the relative size of the element-raw-data and desired image grids. Indeed, the computation time of the operation $\mathrm{H} \gamma$ is relatively longer than the one of $\mathrm{H}^{\star} \boldsymbol{m}$ in the DW case since the image grid is larger than the element-raw-data grid. It is not the case in the PW case where the two grids are the same.

\section{RESUlTS: COMPRESSED BEAMFORMING}

One issue in US imaging, when analog-to-digital conversion (ADC) is carried out in the probe head, is the amount of data that needs to be transferred from the probe to the US system for each insonification. When ADC is carried out in the main system, state-of-the art coaxial cables are not able to embed more than few hundreds coaxial lines, therefore limiting the connections to a few hundreds transducer elements. For probes with a higher number of elements, such as in matrix probes for 3D-imaging, time multiplexing is used which may severely impact the frame rate and the range of application. In this section, we explore how the $\mathrm{CB}$ framework described in Section III-C could alleviate this bottleneck and benefit the 3D-imaging case.

\section{A. A deep dive into coherence}

In order to study US image reconstruction in a CS perspective, the mutual coherence of the matrix $\mathrm{H}_{d} \Psi$, defined in Section III-C, is evaluated for different sampling strategies and sparsifying bases $\Psi$. For computational purposes, only 32 transducer elements are simulated and 256 samples are considered in the axial direction with corresponding depth ranging between $5 \mathrm{~mm}$ and $11 \mathrm{~mm}$. The measurement model considered in the study is based on SPW imaging. The coherence is calculated as the maximum non-diagonal value of the Gram matrix of $\mathrm{H}_{d} \Psi$ [47]. Two different bases $\Psi$ are evaluated, namely the Dirac basis ( $\Psi$ is the identity) and the Haar wavelet (Daubechies 1 wavelet) basis, since we are interested in wavelet-based models. Our choice of wavelet basis is limited to Haar wavelet since it is the only one that has a corresponding matrix expression, making the computation of the mutual coherence feasible. Four different sampling strategies are compared: uniform selection of transducer elements, random selection of transducer elements, CMIX and CTMIX. Regarding CMIX and CTMIX, the matrix W is generated with coefficients distributed according to normal and Rademacher distributions. For CTMIX, the coherence is evaluated for several values of $D_{t}$.

Figures $6 \mathrm{a}$ and $6 \mathrm{~b}$ display the mutual coherence $\mu\left(\mathrm{H}_{d} \Psi\right)$ for a number of measurements ranging between $5 \%$ and $100 \%$, where $\mathrm{H}_{d}$ is square in the case of $100 \%$, with $\Psi$ being Dirac and Haar bases, respectively. It can be seen that the main benefit of the CMIX and CTMIX strategies reside in their ability to limit the increase of the coherence $\mu\left(\mathrm{H}_{d} \Psi\right)$ induced by the undersampling of the raw data. In addition, it can be noticed that this effect is more pronounced for the Haar basis than for the Dirac basis. A comparison between CMIX and CTMIX shows that CTMIX has lower coherence than CMIX. This is expected, since CTMIX provides a better mixing than CMIX.

Regarding the impact of the probability distribution of the random coefficients on $\mu\left(\mathrm{H}_{d} \Psi\right)$, Figure 6c shows that there is no significant difference in coherence between Gaussian and Rademacher random coefficients.

Regarding CTMIX, Figure 6d displays the values of $\mu\left(\mathrm{H}_{d} \Psi\right)$ for the different values of $D_{t}$. It can be seen that the mutual coherence decreases when $D_{t}$ increases. Such a result is consistent with the theoretical analysis carried out in Section III-C2. For $D_{t} \geq 10$, one may observe that the coherence values are rather similar. A finer analysis of the coherence for a number of measurements between $0.11 \%$ and $10 \%$ may exhibit the differences in terms of coherence between the values of $D_{t}$. However, such high number of measurements are not considered in the current study.

\section{B. Reconstruction of the point-reflector phantom}

The B-mode images, displayed on Figure 7 for PW and DW imaging, show that CB (50 iterations), coupled with CMIX, leads to high-quality reconstruction of point sources even for low numbers of measurements (less than $5 \%$ ) for both PW and DW imaging. Two reasons may explain such a result. First, point sources are very sparse, thus well suited to the CS framework. In addition, the measurement matrices associated with CMIX and CTMIX still have a sufficiently low mutual coherence to ensure the perfect recovery condition stated in Theorem 3.1 from [35].

Figure 8 illustrates the peak-signal-to-noise ratio (PSNR) values, calculated on the normalized envelope image (normalized means divided by its maximum value), of the different 


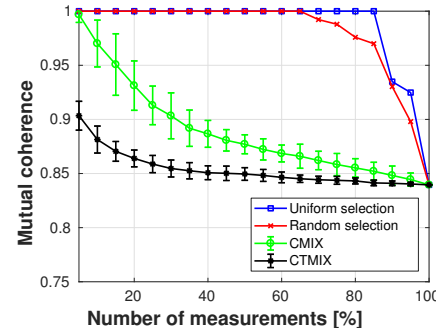

(a)

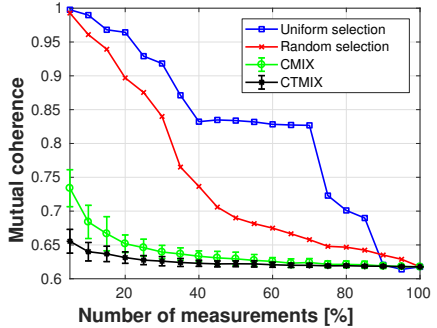

(b)

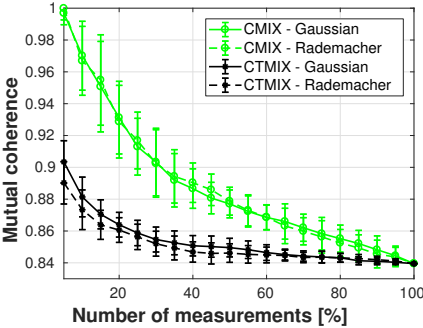

(c)

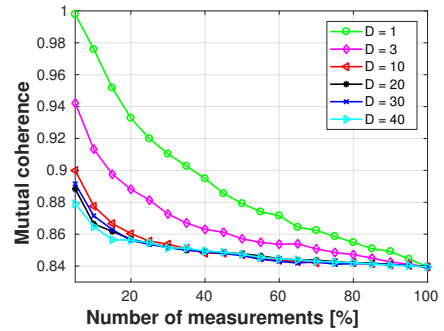

(d)

Fig. 6: Mutual coherence $\mu\left(\mathrm{H}_{d} \Psi\right)$ against the number of measurements for (a) Dirac basis and (b) Haar basis for the uniform selection of transducer elements, the random selection of transducer elements, CMIX and CTMIX $\left(D_{t}=10\right)$. Additionally, the coherence is evaluated (c) for CMIX and CTMIX with mixing coefficients drawn using normal and Rademacher distributions and (d) for CTMIX at different depths.

methods against the reference image, chosen to be the one reconstructed with DAS without data compression.

It can be concluded that CMIX and CTMIX achieve high quality reconstructions for all the considered number of measurements. It can also be observed that, in the case of CMIX, the PSNR drops for numbers of measurements lower than $5 \%$ while it remains constant for CTMIX at lower numbers of measurements. Such results are in agreement with the results on the mutual coherence described in Section VI-A.

Regarding the naive strategy where the undersampling corresponds to the selection of few transducer elements, it can be seen that the PSNR remains high until $10 \%$ measurements and dramatically decreases for lower numbers of measurements. Indeed, because the proposed undersampling strategy is highly coherent, the number of measurements required for perfect reconstruction is higher. Thus, the recovery condition stated by Theorem 3.1 from [35] breaks down for higher numbers of measurements than more incoherent strategies such as CMIX and CTMIX.

\section{Reconstruction of the anechoic phantom}

The CNR values, summarized in Table $\mathrm{V}$ for a number of measurements between $20 \%$ and $50 \%$, show that CTMIX and CMIX outperform existing strategies. It can also be noticed that CMIX and CTMIX leads to similar results, except at $20 \%$ where CTMIX is superior to CMIX.

One may observe a dramatic decrease of the CNR for $20 \%$ measurements. The same coherence argument as for the point-reflector phantom may be used to explain such a fact. Compared to the point-reflector-phantom, the decrease appears at a noticeably higher number of measurement due to the fact that the anechoic inclusion is less sparse than the point-reflector phantom.

\section{Reconstruction of the in vivo carotids}

Figures $9 \mathrm{~b}$ and $9 \mathrm{~d}$ display the B-mode images of the in vivo carotids obtained with CB (1000 iterations), with $20 \%$ measurements. Figures $9 \mathrm{a}$ and $9 \mathrm{c}$ display reference images, reconstructed with DAS without data compression. One may notice that textural areas such as carotid plaques and muscle fibers, as well as anechoic areas, are well reconstructed with
TABLE V: CNR values in $\mathrm{dB}$ for the three undersampling strategies considered and for different numbers of measurements.

\begin{tabular}{|cccc|}
\hline \multirow{2}{*}{ Method } & \multicolumn{3}{c|}{ Nb. of measurements } \\
& $50 \%$ & $25 \%$ & $20 \%$ \\
\hline Uniform selection & 8.81 & 6.03 & 0.57 \\
CMIX & 9.15 & 8.06 & 6.92 \\
CTMIX & 9.16 & 8.10 & 7.30 \\
\hline
\end{tabular}

TABLE VI: Computation times [ms] of the compressed beamforming for the three compression strategies $(25 \%$ measurements) and for the non-compressed case.

\begin{tabular}{|ccc|}
\hline \multirow{2}{*}{ Comp. strategy } & \multicolumn{2}{c|}{ Images } \\
& cyst. & carotid \\
\hline Uniform selection & 87 & 144 \\
CMIX & 130 & 180 \\
CTMIX & 212 & 260 \\
No compression & 119 & 164 \\
\hline
\end{tabular}

CB. However, speckle areas, especially in the far-field, are not well retrieved by the proposed approach, resulting in a darkening of the deepest part of Figures $9 \mathrm{~b}$ and $9 \mathrm{~d}$. This may be explained by the same fact as for SR, i.e. that the waveletbased models do not preserve well the speckle information, which is thus suppressed during the image reconstruction process. It can also be noticed that the number of measurements is higher than in the case of the point-reflector phantom. This is directly linked to differences of the image under scrutiny in terms of sparsity.

Figure 10 displays the PSNR values for different numbers of measurements, ranging between $14 \%$ and $50 \%$. One may observe the same behaviour as for the point-reflector phantom. CB achieves a high quality reconstruction even for low numbers of measurements, allowing a drastic reduction in the required number of measurements, for each insonification.

\section{E. Computation times for compressed beamforming}

The computation times for the compressed beamforming (50 iterations of PDFB, 25\% measurements) are summarized in Table VI. The proposed methods, as for the sparse regularization for image enhancement method described in Section III-B, are three orders of magnitude faster than existing compressed beamforming methods [10]. Regarding the timing of the 


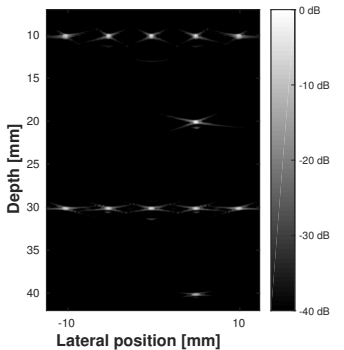

(a)

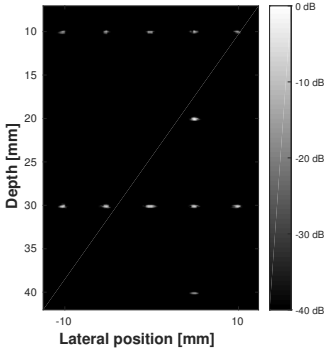

(b)

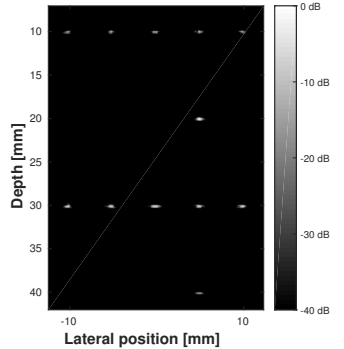

(c)

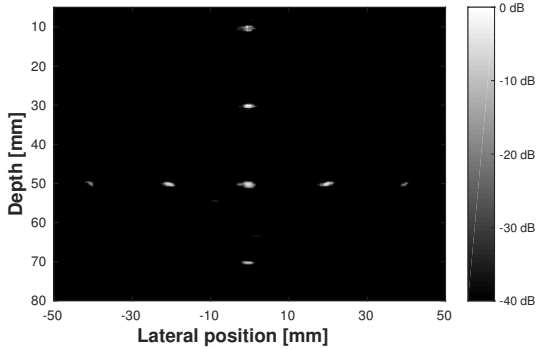

(d)

Fig. 7: B-mode images of the point-reflector phantom for $1 \mathrm{PW}$ insonification ((a) and (b)) and 1 DW insonification ((c) and (d)) reconstructed with DAS ((a) and (c)) and with compressed beamforming coupled with CMIX strategy with $4 \%$ measurements ((b) and (d)).

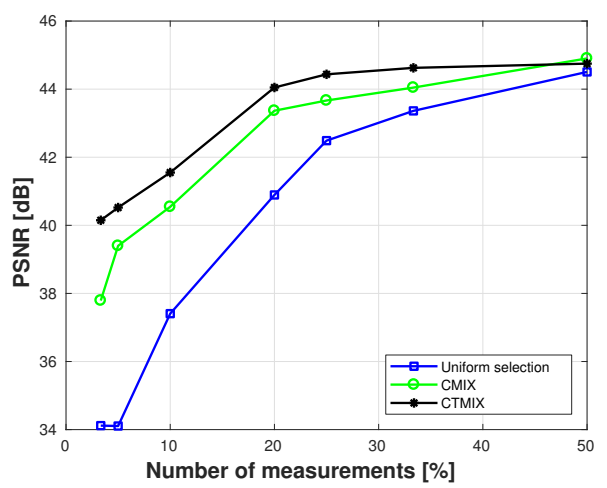

Fig. 8: PSNR against the number of measurements for the point-reflector phantom insonified with 1 PW for CTMIX, CMIX and the uniform selection of transducer elements.

different strategies, the fastest one is the uniform selection. This makes sense since no additional matrix multiplication is achieved. Regarding CMIX and CTMIX, the multiplication with the random matrix leads to a significant increase of the computation times, which are higher than in the noncompressed case, as shown on Table VI. In addition, the compressed beamforming method coupled with CMIX and CTMIX is no longer matrix-free since the random matrices have to be stored in memory. Thus, the CMIX and CTMIX strategies may be of interest if they come with a simplification of the hardware, at the cost of a more computationally demanding reconstruction. These points will be discussed in Section VII-B.

\section{DISCUSSION}

A. Towards real-time sparse regularization for enhanced image reconstruction

In this work, we have leveraged the problem of memory footprint, which is one of the most challenging aspects in US image reconstruction by $\mathrm{SR}$, as stated in the previous works mentioned in Section I. We have shown that, by exploiting the high parallelization potential of the measurement model, its adjoint and by using fast wavelet transforms [48], the proposed method may achieve image reconstruction two to three orders of magnitude faster than state-of-the-art SR approaches, with minimal memory requirements. This paves the way to the extension of SR to 3D- imaging, which has not been possible with state-of-the-art methods due to the problem of matrix coefficients storage.

However, the computation times presented in the study are not compatible with real-time imaging yet, mainly due to the computational complexity of the proposed model. The computations time reported in the proposed work are based on a non optimized-code. Thus, one way to accelerate the proposed method consists in optimizing the implementation of the forward model and the adjoint, especially by minimizing the data transfer between the CPU and the GPU. In addition, with the explosion of the computational power of GPUs in the last years, it can be expected that the computation times may be one order of magnitude lower with the next generation of GPUs. Another way towards real-time applications is to lower the number of iterations necessary to reach a given image quality. This can be performed by a better tuning of the hyperparameters. One recent trend is to consider the use of deepneural networks in order to tune the hyper-parameters based on a training set. This technique has given very promising results on 1D-signals [49].

The advantage of having a real-time SR is twofold. Firstly, it provides an image enhancement technique, especially for the detection of anechoic area. Secondly, it allows for a reduction of the number of insonifications necessary to reach a given image quality, which is crucial in US imaging application where power supply and power dissipation are problematic, e.g. in portable systems.

\section{B. Towards compressed sensing in ultrasound imaging}

In the proposed work, we suggest innovative sampling schemes for US imaging and thus go one step further towards CS in US image reconstruction. Indeed, CMIX and CTMIX strategies outperform existing compression strategies, mainly based on selection of transducer elements, in terms of image quality, as demonstrated in Section VI. However, we face one major obstacle which is the high coherence of the measurement operator. CMIX and CTMIX strategies manage to maintain the coherence constant when the number of measurements is decreased, but the coherence remains high relative to the corresponding Welch bound, because of the 


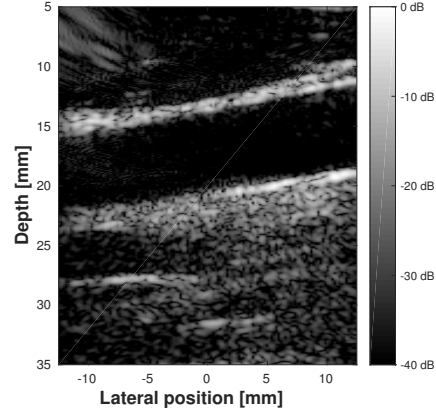

(a)

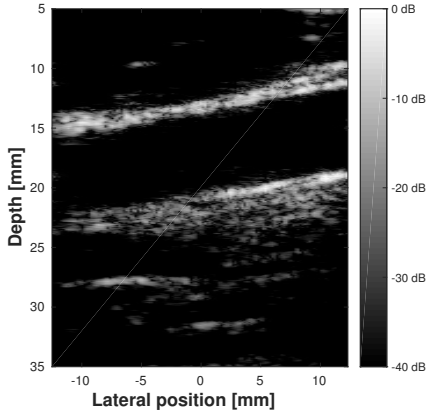

(b)

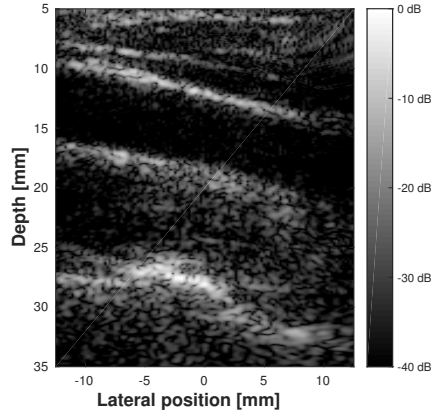

(c)

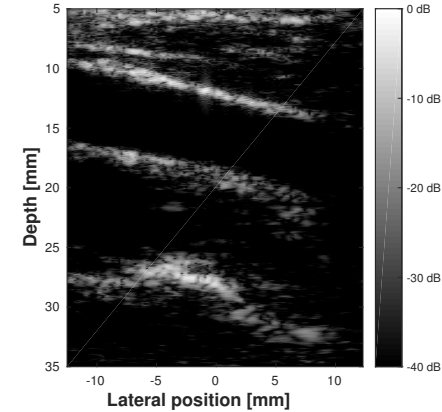

(d)

Fig. 9: B-mode images of the in vivo carotids for $1 \mathrm{PW}$ insonification reconstructed with DAS ((a) and (c)) and with the compressed beamforming ((b) and (d)) coupled with the CMIX strategy and $20 \%$ measurements.

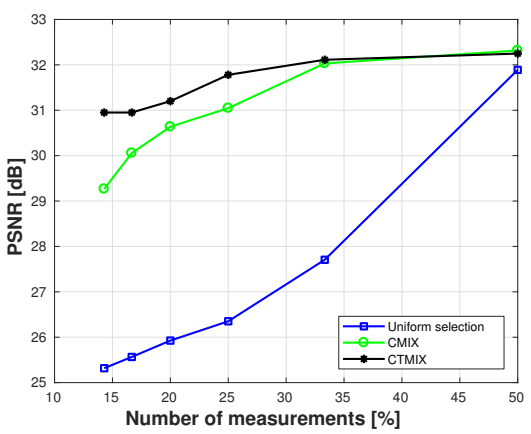

Fig. 10: PSNR against the number of measurements for the in vivo carotid insonified with $1 \mathrm{PW}$ and sampled for CTMIX, CMIX and the uniform selection of transducer elements.

coherence intrinsic to the measurement model. Indeed, the high mutual coherence of the measurement model comes from the fact that each projection onto a 1D-conic, implied by the time-of-flight calculations, involves only a few points in the desired-image space. The natural question that one may ask is whether it is possible to change the nature of the projections in order to involve more points. In fact, this is a relatively difficult task since projections are a consequence of the expression for the round-trip time-of-flight of US waves in a homogeneous medium. This observation has a deep impact on the design of CS acquisition schemes. Indeed, it means that the attempts to decrease the coherence of the measurement model by playing with random pulses sent in transmit is hopeless, because this does not change the expression for the time-of-flight, and thus the fact that echo-samples stem from projections onto 1Dconics.

One solution to this issue may reside in dealing with the coherent operator, by exploring a recent topic on CS, denoted "constrained adaptive sensing", which derives sampling theorems and variants of Theorem 3.1 from [35] where the measurement matrix is more constrained than in standard CS [50].

Another alternative could be to entirely rethink the measurement process, starting from the requirements of Theorem 3.1 from [35]. Let us consider that we are in a case where the matrix $\mathrm{H}$ is random, which is compatible with the requirements of Theorem 3.1 from [35]. In terms of acoustic propagation, a random measurement model implies that each sample of the element raw-data receives contributions from points spread over the entire image space. In other words, it means that the duality between time and depth, which is at the heart of US imaging, is not valid anymore since echoes from points at different positions reach the transducer elements at the same time. Thus, a random measurement model $\mathrm{H}$ is unfeasible, in pulse-echo imaging in a homogeneous medium, due to the fact that US waves respect the Helmholtz equation. One way to address such an issue resides in placing a scattering or heterogeneous medium in front of the US probe. This principle has been recently developed in optics and gives promising results [51]. However, such a new design raises many questions regarding the choice and the modelling of the heterogeneous medium. It can be easily understood that such a medium may not generate a purely random matrix but will be somewhere inbetween a purely random case and the highly coherent case of the homogeneous medium. This topic is currently under study and will be the object of further reports or communications.

\section{Limitations of sparsity-driven image reconstruction meth- ods in ultrafast ultrasound imaging}

1) The sparsity prior: The first limitation inherent to iterative methods lies in the regularization term. Indeed, the prior knowledge on the signal under scrutiny, especially when it comes to imaging living bodies, cannot cover all the cases that one may encounter. Taking that into account, two solutions may be considered.

The first one consists in using analytical models, such as wavelet-based models, which perform well on a wide range of images. This approach is the one we used in this study as well as in our previous work [14]. However, even if waveletbased models achieve good performance, they tend to decrease the speckle density compared to DAS.

The other alternative consists in using dictionaries, learned on the data by means of dictionary learning algorithms. This has been recently applied to US image reconstruction [21]. At first sight, such a technique appears to be particularly well suited to US imaging. Indeed, US probes are, most of the time, dedicated to one specific application and work at a given frequency. This allows to restrict the size of the training set and facilitates the learning procedure. However, such types 
of prior suffer from two major drawbacks. The first one is the risk of overfitting. Indeed, it is a hard task to build a training set of US images whose quality depends on an infinite variety of parameters such as the positioning of the probe, the patient anatomy, etc. In addition, having a sparse prior in a dictionary prevents using fast transforms, which makes its use more difficult in a real-time environment.

2) Sensitivity to the hyper-parameters and data dependency: The efficiency of iterative methods, compared to analytical approaches, depends on the data that one may want to reconstruct because of the sparsity prior and the hyper-parameters intrinsic to the optimization algorithm. In this work, as well as in previous studies [10], [14], [33], it is firstly shown that SR methods perform well on sparse sources, which make them attractive for harmonic imaging of microbubbles. This can be easily explained by the fact that point sources are very sparse. Secondly, SR methods are efficient for reconstructing images made of structured fibers and anechoic areas such as large vessels, heart cavities, etc. Indeed, such methods manage to remove the noise present in anechoic areas which tends to reinforce the relative intensity of the structured parts. However, these methods struggle when it comes to imaging hyperechoic area. They put too much emphasis on the hyperechoic part to the detriment of the background as described in our previous work [14].

Regarding $\mathrm{CB}$, we observe that, when mixing element raw-data arising from a hyperechoic region embedded in a less echogeneous background, the high intensities of the hyperechoic region tend to interfere with the lower intensity regions, which makes the resolution of the inverse problem very difficult. We also notice that $\mathrm{CB}$ is even more sensitive to the sparsity prior and the hyper-parameters than the SR approach used for enhanced image reconstruction, when a mixing is performed. This can be explained by the fact that the inverse problem involving the mixing is more difficult to solve than the one posed by SR for enhanced image reconstruction.

One solution to the problem of the hyper-parameters may be to investigate deep-learning approaches, which have been successfully used to solve sparse-coding problems [52], [49]. The idea consists in mapping one iteration of a convex optimization to one layer of a deep neural network (DNN). The hyperparameters as well as the non-linearities are learned during the training process of the DNN. The resulting DNN has shown promising results in terms of number of iterations required for convergence and reconstruction quality and is currently under study for the proposed problem. Another solution may consist in investigating in depth the noise induced by the proposed model. While the noise implied by the choice of the proposed model seems to be difficult to quantify, the noised induced by the discretization of the proposed model, i.e. by the interpolation of the continuous integral onto the discrete grid may be studied in depth, in order to identify a lower bound for $\epsilon$.

\section{CONCLUSION}

The proposed work presents novel parametric, fast and matrix-free formulations of the measurement model and its adjoint in the context of ultrafast ultrasound imaging. These formulations are included in a sparse regularization framework which is used to achieve high-quality imaging three orders of magnitude faster than existing methods. By exploiting a sparsity prior of ultrasound images in a model made of a concatenation of wavelets, it outperforms both the delay-andsum algorithm and the best existing methods, as demonstrated on simulation and in vivo experiments. In addition, new undersampling strategies, more suited to compressed sensing than previous ones, are suggested and high quality reconstruction with a low number of measurements are demonstrated. By coupling fast measurement operators suitable for GPU implementation with efficient regularization methods, this work paves the way for enhanced ultrafast ultrasound imaging in both 2D and 3D. It also explores, questions and suggests new concepts for the application of CS to ultrasound image reconstruction, based on its fundamental principles.

\section{ACKNOWLEDGMENT}

This work was supported in part by the UltrasoundToGo RTD project (no. 20NA21_145911), evaluated by the Swiss NSF and funded by Nano-Tera.ch with Swiss Confederation financing. This work was also supported by the UK Engineering and Physical Sciences Research Council (EPSRC, grants EP/M019306/1). The RF Verasonics system was co-funded by the FEDER program, Saint-Etienne Metropole (SME) and Conseil General de la Loire (CG42) within the framework of the SonoCardio-Protection Project lead by Prof. Pierre Croisille. The authors would also like to thank Dr Olivier Bernard from CREATIS laboratory for providing the in vivo acquisition data.

\section{APPENDIX A: DERIVATION OF THE PARAMETRIC EQUATIONS FOR A STEERED PLANE WAVE AND A DIVERGING WAVE}

Let us remind the forward problem defined in Equation (8):

$$
h\left(x_{t}, t\right)=\int_{\boldsymbol{r} \in \Gamma\left(x_{t}, t\right)} \frac{o_{d}\left(\boldsymbol{r}, x_{t}\right) \gamma(\boldsymbol{r})}{\left|\nabla_{\boldsymbol{r}} g\right|} d \sigma(\boldsymbol{r}) \text {, }
$$

in which $g\left(\boldsymbol{r}, x_{t}, t\right)=t-t_{T x}(\boldsymbol{r})-t_{R x}\left(\boldsymbol{r}, x_{t}\right)$, and $\Gamma\left(x_{t}, t\right)=$ $\left\{\boldsymbol{r} \in \Omega \mid g\left(\boldsymbol{r}, x_{t}, t\right)=0\right\}$.

\section{A. Steered plane waves}

Let us consider a SPW with angle $\theta$. As shown by Montaldo et al. [41], the propagation delay in transmit can be written as:

$$
t_{T x}(x, z)=\frac{x-x_{t}^{\star}}{c} \sin \theta+\frac{z}{c} \cos \theta
$$

where $x_{t}^{\star}$ is the position of the transducer element which emits first. 
According to Equation (29), the following equivalence holds:

$$
\begin{aligned}
\boldsymbol{r} & =[x, z]^{T} \in \Gamma\left(x_{t}, t\right) \\
& \Leftrightarrow \sqrt{\left(x-x_{t}\right)^{2}+z^{2}}+\left(x-x_{t}^{\star}\right) \sin \theta+z \cos \theta=c t \\
& \Leftrightarrow\left(c t-\left(x-x_{t}^{\star}\right) \sin \theta-z \cos \theta\right)^{2}-\left(x-x_{t}\right)^{2}-z^{2}=0 \\
& \Leftrightarrow A z^{2}+B z+C=0 \\
\Leftrightarrow z & \Leftrightarrow \frac{-B \pm \sqrt{\Delta}}{2 A} .
\end{aligned}
$$

where $\Delta=B^{2}-4 A C$ and $A, B$ and $C$ are defined by:

$$
\left\{\begin{array}{l}
A=-\sin ^{2} \theta, \\
B=2\left(x-x_{t}^{\star}\right) \cos \theta \sin \theta-2 c t \cos \theta, \\
C=\left(c t-\left(x-x_{t}^{\star}\right) \sin \theta\right)^{2}-\left(x-x_{t}\right)^{2} .
\end{array}\right.
$$

In the specific case where $A=0$, i.e. in the case where $\theta=0$, the parametric equation can be directly expressed as:

$$
z\left(x, x_{t}, t\right)=\frac{1}{2 c t}\left((c t)^{2}-\left(x-x_{t}\right)^{2}\right) .
$$

\section{B. Diverging waves}

Let us consider a DW with a virtual point source located at $\left[x_{n}, z_{n}\right]^{T}$. As demonstrated by Papadacci et al. [42], the propagation delay in transmit can be written as:

$$
t_{T x}(x, z)=\sqrt{\left(x-x_{n}\right)^{2}+\left(z-z_{n}\right)^{2}}-d_{0},
$$

where $d_{0}=\min _{x_{t}} \sqrt{\left(x_{n}-x_{t}\right)^{2}+z_{n}^{2}}$.

According to Equation (33), the following equivalence holds:

$$
\begin{aligned}
& \boldsymbol{r}=[x, z]^{T} \in \Gamma\left(x_{t}, t\right) \\
& \Leftrightarrow 4\left(\left(x-x_{n}\right)^{2}+\left(z-z_{n}\right)^{2}\right)\left(\left(x-x_{t}\right)^{2}+z^{2}\right)= \\
& \left(\left(c t+d_{0}\right)^{2}-\left(x-x_{n}\right)^{2}-\left(z-z_{n}\right)^{2}-\left(x-x_{t}\right)^{2}-z^{2}\right)^{2} \\
& \Leftrightarrow(A+z)^{2}=B \\
& \Leftrightarrow z=-A \pm \sqrt{B},
\end{aligned}
$$

where

$$
A=\frac{-z_{n}}{2}\left(1+\frac{\left(x_{t}-x\right)^{2}-\left(x_{n}-x\right)^{2}}{\left(d_{0}+c t\right)^{2}-z_{n}^{2}}\right) \text {, }
$$

and

$$
\begin{gathered}
B=\frac{\left(d_{0}+c t\right)^{2}}{4\left(z_{n}^{2}-\left(d_{0}+c t\right)^{2}\right)^{2}}\left(\left(x_{t}-x_{n}\right)^{2}+z_{n}^{2}-\left(d_{0}+c t\right)^{2}\right) \\
\left(\left(x_{t}+x_{n}\right)^{2}+z_{n}^{2}-\left(d_{0}+c t\right)^{2}-4 x\left(x_{t}+x_{n}-x\right)\right) .
\end{gathered}
$$

APPENDIX B: DETAILED MATRIX-FREE FORMULATIONS OF THE MEASUREMENT MODEL AND ITS ADJOINT

\section{A. Measurement model}

Let us remind Equation (9):

$$
\begin{aligned}
h\left(x_{t}, t\right)=\int_{x \in \mathbb{R}} \frac{o_{d}\left(x, z\left(x, x_{t}, t\right), x_{t}\right)}{\left|\nabla_{\left(x, z\left(x, x_{t}, t\right)\right)} g\right|} & \gamma\left(x, z\left(x, x_{t}, t\right)\right)\left|J_{z}(x)\right| d x .
\end{aligned}
$$

If we consider the image grid defined in Section II-A and the interpolating kernel $\varphi: \mathbb{R} \rightarrow \mathbb{R}$, then, according to [53], $\gamma\left(x, z\left(x, x_{t}, t\right)\right)$ can be expressed as:

$$
\begin{array}{r}
\gamma\left(x, z\left(x, x_{t}, t\right)\right)=\sum_{q=1}^{N_{z}} \varphi\left(z^{q}-z\left(x, x_{t}, t\right)\right) \gamma\left(x, z^{q}\right) \\
+\nu_{\varphi}\left(z\left(x, x_{t}, t\right), \boldsymbol{z}\right)
\end{array}
$$

where $\nu_{\varphi}\left(z\left(x, x_{t}, t\right), \boldsymbol{z}\right) \in \mathbb{R}$ accounts for the interpolation error. The discretization of Equation (9) leads to the following formulation:

$$
\begin{array}{r}
h\left(x_{t}, t\right)=\sum_{n=1}^{N_{x}} \frac{o_{d}\left(x^{n}, z\left(x^{n}, x_{t}, t\right), x_{t}\right)\left|J_{z}\left(x^{n}\right)\right|}{\left|\nabla_{\left(x^{n}, z\left(x^{n}, x_{t}, t\right)\right)}\right|} \\
\sum_{q=1}^{N_{z}} \varphi\left(z^{q}-z\left(x^{n}, x_{t}, t\right)\right) \gamma\left(x^{n}, z^{q}\right) \\
\quad+\nu_{\varphi}\left(z\left(x^{n}, x_{t}, t\right), z^{q}\right) .
\end{array}
$$

To end up with the complete formulation of the measurement model, we deduce from Equation (7) that $m\left(x_{t}, t\right)=$ $\left(h\left(x_{t}\right) *_{t} v_{p e}\right)(t)$, where $*_{t}$ denotes the 1D-discrete convolution, which can be easily implemented "on-the-fly".

Thus, the element-raw data are related to the TRF samples by the following operator:

$$
\begin{aligned}
& \mathcal{H}\left(\gamma, x_{t}, t\right)=\nu_{\varphi}^{\prime}\left(x_{t}, t\right)+\sum_{l=1}^{N_{t}} v_{p e}\left(t-t^{l}\right) \\
& \sum_{n=1}^{N_{x}} \frac{o_{d}\left(x^{n}, z\left(x^{n}, x_{t}, t^{l}\right), x_{t}\right)\left|J_{z}\left(x^{n}\right)\right|}{\left|\nabla_{\left(x^{n}, z\left(x^{n}, x_{t}, t^{l}\right)\right)}\right|} \\
& \sum_{q=1}^{N_{z}} \varphi\left(z^{q}-z\left(x^{n}, x_{t}, t^{l}\right)\right) \gamma\left(x^{n}, z^{q}\right),
\end{aligned}
$$

which can be computed "on-the-fly" and in parallel for each point of the element-raw-data grid and in which $\nu_{\varphi}^{\prime}\left(x_{t}, t\right)$ is the overall error defined by:

$$
\begin{gathered}
\nu_{\varphi}^{\prime}\left(x_{t}, t\right)=\sum_{l=1}^{N_{t}} v_{p e}\left(t-t^{l}\right) \\
\sum_{n=1, q=1}^{N_{x}, N_{z}} \frac{o_{d}\left(x^{n}, z\left(x^{n}, x_{t}, t^{l}\right), x_{t}\right)\left|J_{z}\left(x^{n}\right)\right|}{\mid \nabla_{\left(x^{n}, z\left(x^{n}, x_{t}, t^{l}\right)\right)^{g} \mid}} \nu_{\varphi}\left(z\left(x^{n}, x_{t}, t^{l}\right), z^{q}\right) .
\end{gathered}
$$

Thus, by evaluating Equation (40) for each point $\left(x_{t}^{j}, t^{l}\right)$ of the element-raw-data grid, the following relationship holds:

$$
m\left(x_{t}^{j}, t^{l}\right)=\mathcal{H}\left(\gamma, x_{t}^{j}, t^{l}\right)+\nu_{\varphi}^{\prime}\left(x_{t}^{j}, t^{l}\right)
$$

which defines Equation (10) by taking into account the other sources of noise (model and measure). 


\section{B. Adjoint operator of the measurement model}

The adjoint operator of the measurement model is defined in Equation (11) as:

$$
\begin{aligned}
\hat{\gamma}(\boldsymbol{r})= & \int_{x_{t} \in \Xi} o_{d}\left(\boldsymbol{r}, x_{t}\right) \\
& \left(m\left(x_{t}\right) *_{t} u\right)\left(t_{T x}(\boldsymbol{r})+t_{R x}\left(\boldsymbol{r}, x_{t}\right)\right) d x_{t} .
\end{aligned}
$$

If we set $t\left(\boldsymbol{r}, x_{t}\right)=t_{T x}(\boldsymbol{r})+t_{R x}\left(\boldsymbol{r}, x_{t}\right)$, we consider the element-raw data grid defined in Section II-A and the interpolating kernel $\varphi: \mathbb{R} \rightarrow \mathbb{R}$, then, similarly to Equation (38), $m\left(x_{t}, t\left(\boldsymbol{r}, x_{t}\right)\right)$ can be expressed as:

$$
\begin{aligned}
m\left(x_{t}, t\left(\boldsymbol{r}, x_{t}\right)\right)=\sum_{l=1}^{N_{t}} \varphi\left(t\left(\boldsymbol{r}, x_{t}\right)\right. & \left.-t^{l}\right) m\left(x_{t}, t^{l}\right) \\
& +\xi_{\varphi}\left(t\left(\boldsymbol{r}, x_{t}\right), \boldsymbol{t}\right),
\end{aligned}
$$

where $\xi_{\varphi}\left(t\left(\boldsymbol{r}, x_{t}\right), \boldsymbol{t}\right) \in \mathbb{R}$ accounts for the interpolation error.

The discretization of Equation (11) leads to the following formulation:

$$
\begin{aligned}
\mathcal{H}^{\star}(\boldsymbol{m}, \boldsymbol{r})= & \xi_{\varphi}^{\prime}(\boldsymbol{r})+\sum_{l=1}^{N_{t}} u\left(t^{l}\right) \sum_{j=1}^{N_{e l}} o_{d}\left(\boldsymbol{r}, x_{t}^{j}\right) \\
& \sum_{s=1}^{N_{t}} \varphi\left(t\left(\boldsymbol{r}, x_{t}^{j}\right)-t^{l}-t^{s}\right) m\left(x_{t}^{j}, t^{s}\right),
\end{aligned}
$$

which can be computed "on-the-fly" and in parallel for each point of the image grid and in which $\xi_{\varphi}^{\prime}(\boldsymbol{r})=$ $\sum_{l=1, j=1, s=1}^{N_{t}, N_{e l}, N_{t}} u\left(t^{l}\right) o_{d}\left(\boldsymbol{r}, x_{t}^{j}\right) \xi_{\varphi}\left(t\left(\boldsymbol{r}, x_{t}^{j}\right)-t^{l}, t^{s}\right)$.

Thus, by evaluating Equation (45) for each point $\left(x^{n}, z^{q}\right)$ of the image grid, the following relationship holds:

$$
\gamma\left(x^{n}, z^{q}\right)=\mathcal{H}^{\star}\left(\boldsymbol{m}, x^{n}, z^{q}\right)+\xi_{\varphi}^{\prime}\left(x^{n}, z^{q}\right),
$$

which defines Equation (12) by taking into account the other sources of noise (model and measure).

\section{APPENDIX C: AdJOINT OPERATOR OF THE CONTINUOUS MEASUREMENT MODEL}

The continuous measurement model, defined in Equation (2), can be written as:

$$
m\left(x_{t}, t\right)=\mathcal{T}\{\gamma\}\left(x_{t}, t\right),
$$

where $\mathcal{L}_{2}(\Omega)$ designates the space of square integrable functions with values in $\Omega$ and $\mathcal{T}: \mathcal{L}_{2}(\Omega) \rightarrow \mathcal{L}_{2}(\Xi \times \mathbb{R})$ is a functional described by:

$$
\begin{aligned}
\mathcal{T}\{\gamma\}\left(x_{t}, t\right)= & \int_{\boldsymbol{r} \in \Omega} \gamma(\boldsymbol{r}) o_{d}\left(\boldsymbol{r}, x_{t}\right) \\
& v_{p e}\left(t-t_{T x}(\boldsymbol{r})-t_{R x}\left(\boldsymbol{r}, x_{t}\right)\right) d \boldsymbol{r} .
\end{aligned}
$$

In order to define the adjoint of the operator $\mathcal{T}$, let us introduce a function $n \in \mathcal{L}_{2}(\Xi \times \mathbb{R})$. The inner product between $\mathcal{T}\{\gamma\}$ and $n$ can be written as:

$$
<\mathcal{T}\{\gamma\}, n>=\iint_{x_{t} \in \Xi, t \in \mathbb{R}} \mathcal{T}\{\gamma\}\left(x_{t}, t\right) n\left(x_{t}, t\right) d x_{t} d t,
$$

which can be expressed as:

$$
\begin{aligned}
& <\mathcal{T}\{\gamma\}, n>=\iint_{\boldsymbol{r} \in \Omega, x_{t} \in \Xi} \gamma(\boldsymbol{r}) o_{d}\left(\boldsymbol{r}, x_{t}\right) \\
& \int_{t \in \mathbb{R}} n\left(x_{t}, t\right) v_{p e}\left(t-t_{T x}(\boldsymbol{r})-t_{R x}\left(\boldsymbol{r}, x_{t}\right)\right) d x_{t} d t d \boldsymbol{r} .
\end{aligned}
$$

Let us now focus on the integral over the variable $t$ which can be formulated as:

$$
\begin{aligned}
& \int_{t \in \mathbb{R}} n\left(x_{t}, t\right) v_{p e}\left(t-t_{T x}(\boldsymbol{r})-t_{R x}\left(\boldsymbol{r}, x_{t}\right)\right) d x_{t} d t \\
& =\int_{t \in \mathbb{R}} n\left(x_{t}, t\right) u\left(t_{T x}(\boldsymbol{r})+t_{R x}\left(\boldsymbol{r}, x_{t}\right)-t\right) d x_{t} d t \\
& =\left(n\left(x_{t}\right) *_{t} u\right)\left(t_{T x}(\boldsymbol{r})+t_{R x}\left(\boldsymbol{r}, x_{t}\right)\right),
\end{aligned}
$$

where $u(t)=v_{p e}(-t)$ is the matched filter of the pulse-echo waveform.

This leads to the following expression for the inner product:

$$
\begin{aligned}
&<\mathcal{T}\{\gamma\}, n>= \int_{\boldsymbol{r} \in \Omega} \gamma(\boldsymbol{r}) \int_{x_{t} \in \Xi} o_{d}\left(\boldsymbol{r}, x_{t}\right) \\
&\left(n\left(x_{t}\right) *_{t} u\right)\left(t_{T x}(\boldsymbol{r})+t_{R x}\left(\boldsymbol{r}, x_{t}\right)\right) d x_{t} d \boldsymbol{r} .
\end{aligned}
$$

Let us define the operator $\mathcal{T}^{\star}$ from Equation (52) as:

$$
\begin{aligned}
\mathcal{T}^{\star}\{n\}(\boldsymbol{r})= & \int_{x_{t} \in \Xi} o_{d}\left(\boldsymbol{r}, x_{t}\right) \\
& \left(n\left(x_{t}\right) *_{t} u\right)\left(t_{T x}(\boldsymbol{r})+t_{R x}\left(\boldsymbol{r}, x_{t}\right)\right) d x_{t} .
\end{aligned}
$$

Introducing $\mathcal{T}^{\star}$ in Equation (52) leads us to the following equality:

$$
<\mathcal{T}\{\gamma\}, n>=<\gamma, \mathcal{T}^{\star}\{n\}>,
$$

which defines the adjoint operator of $\mathcal{T}$.

\section{APPENDIX D: PRIMAL-DUAL-FORWARD-BACKWARD ALGORITHM}

The general problem we solve, which can be seen as an instance of Problem (12) of [34], is the following one:

$$
\min _{\boldsymbol{x} \in \mathbb{C}^{N}} f_{1}(\boldsymbol{x})+f_{2}(\boldsymbol{\Phi} \boldsymbol{x}-\boldsymbol{y}),
$$

given the assumption that $f_{1}: \mathbb{C}^{N} \rightarrow \mathbb{R}$ and $f_{2}: \mathbb{C}^{M} \rightarrow \mathbb{R}$ are lower semicontinuous convex functions.

The key mathematical tool used in PDFB is the proximity operator of a convex function defined as:

$$
\operatorname{prox}_{f}(\boldsymbol{x})=\arg \min _{\boldsymbol{z} \in \mathbb{C}^{M}} f(\boldsymbol{z})+\frac{1}{2}\|\boldsymbol{z}-\boldsymbol{x}\|_{2}^{2} .
$$

In the proposed $\ell_{1}$-minimization problem $f_{1}(\boldsymbol{x})=\left\|\Psi^{\dagger} \boldsymbol{x}\right\|_{1}$ and $f_{2}(\boldsymbol{x})=i_{B}(\boldsymbol{x})$, where $i_{B}$ is the indicator function of the convex set $B$ defined as $B=\left\{\boldsymbol{x} \in \mathbb{C}^{M} \mid\|\boldsymbol{x}\|_{2} \leq \epsilon\right\}$. 
The general structure of the algorithm is detailed in Algorithm 1. It involves two non-linear operations, the softthresholding denoted as $\operatorname{soft}(\boldsymbol{x} ; \lambda)$ and defined for each element $x_{i}$ of the vector $\boldsymbol{x}$ as $\operatorname{sign}\left(x_{i}\right) \max \left(\left|x_{i}\right|-\lambda, 0\right)$, and the projection on the $\ell_{2}$-ball of radius $\epsilon$ denoted as $\operatorname{proj}_{B}(\boldsymbol{x})=$ $\boldsymbol{x} \min \left(\epsilon /\|\boldsymbol{x}\|_{2}, 1\right)$. The parameters $\sigma_{1}=1, \sigma_{2}=1 / L$, where $L$ is the spectral norm of the matrix $\Phi$, and $\tau=0.5$ are step sizes, and $\gamma>0$ is a thresholding constant that controls the convergence speed.

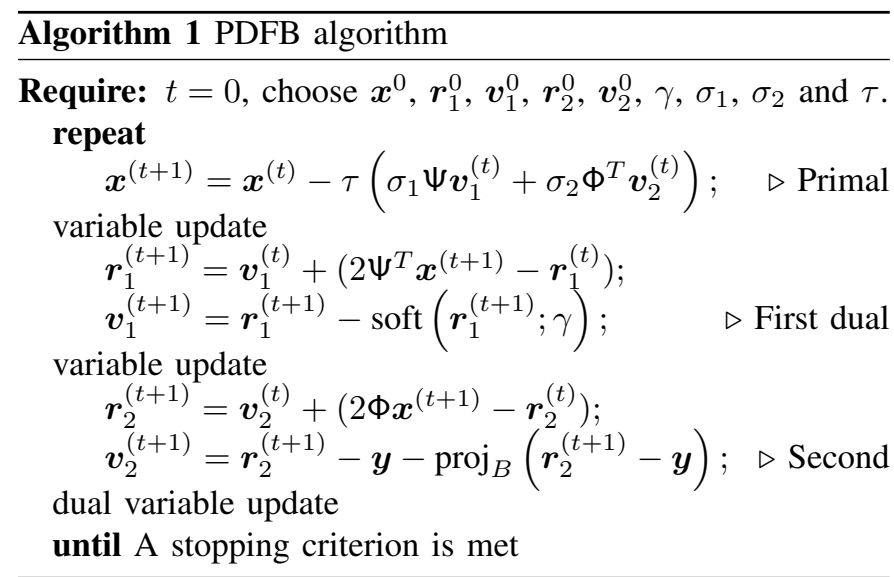

\section{REFERENCES}

[1] M. Tanter and M. Fink, "Ultrafast imaging in biomedical ultrasound," IEEE Trans. Ultrason. Ferroelectr. Freq. Control, vol. 61, no. 1, pp. 102-119, 2014.

[2] N. Ozmen, R. Dapp, M. Zapf, H. Gemmeke, N. V. Ruiter, and K. W. A. van Dongen, "Comparing different ultrasound imaging methods for breast cancer detection," IEEE Trans. Ultrason. Ferroelectr. Freq. Control, vol. 62, no. 4, pp. 637-646, 2015.

[3] T. L. Szabo, Diagnostic Ultrasound Imaging: Inside Out, second edition. Elsevier, 2014.

[4] T. Chernyakova and Y. Eldar, "Fourier-domain beamforming: The path to compressed ultrasound imaging," IEEE Trans. Ultrason. Ferroelectr. Freq. Control, vol. 61, no. 8, pp. 1252-1267, 2014.

[5] O. Scherzer, M. Grasmair, H. Grossauer, M. Haltmeier, and F. Lenzen, Variational methods in imaging. Springer, 2009, vol. 320.

[6] M. Lustig, D. Donoho, and J. M. Pauly, "Sparse MRI: The application of compressed sensing for rapid MR imaging." Magn. Reson. Med., vol. 58, no. 6, pp. 1182-95, 2007.

[7] J. Hsieh, Computed Tomography, Second Edition. 1000 20th Street, Bellingham, WA 98227-0010 USA: SPIE, 2009.

[8] G.-H. Chen, J. Tang, and S. Leng, "Prior image constrained compressed sensing (PICCS): a method to accurately reconstruct dynamic CT images from highly undersampled projection data sets." Med. Phys., vol. 35, no. 2, pp. 660-663, 2008.

[9] E. Y. Sidky and X. Pan, "Image reconstruction in circular cone-beam computed tomography by constrained, total-variation minimization," Phys. Med. Biol., vol. 53, no. 17, pp. 4777-4807, 2008.

[10] G. David, J.-L. Robert, B. Zhang, and A. F. Laine, "Time domain compressive beam forming of ultrasound signals," J. Acoust. Soc. Am., vol. 137, no. 5, pp. 2773-2784, 2015.

[11] Congzhi Wang, Xi Peng, Dong Liang, and Hairong Zheng, "Plane-wave ultrasound imaging based on compressive sensing with low memory occupation," in IEEE Int. Ultrason. Symp., 2015, pp. 1-4.

[12] A. Besson, R. E. Carrillo, O. Bernard, Y. Wiaux, and J.-P. Thiran, "Compressed delay-and-sum beamforming for ultrafast ultrasound imaging," in IEEE Int. Conf. Image Process., 2016, pp. 2509-2513.

[13] B. Zhang, J.-L. Robert, and G. David, "Dual-domain compressed beamforming for medical ultrasound imaging," in IEEE Int. Ultrason. Symp., 2015.

[14] A. Besson, M. Zhang, F. Varray, H. Liebgott, D. Friboulet, Y. Wiaux, J.-P. Thiran, R. E. Carrillo, and O. Bernard, "A sparse reconstruction framework for Fourier-based plane-wave imaging," IEEE Trans. Ultrason. Ferroelectr. Freq. Control, vol. 63, no. 12, pp. 2092-2106, 2016.
[15] M. F. Schiffner and G. Schmitz, "Fast pulse-echo ultrasound imaging employing compressive sensing," IEEE Int. Ultrason. Symp., 2011.

[16] O. Michailovich and A. Tannenbaum, "Despeckling of medical ultrasound images," IEEE Trans. Ultrason. Ferroelectr. Freq. Control, vol. 53, no. 1, pp. 64-78, 2006.

[17] P. C. Tay, C. D. Garson, S. T. Acton, and J. A. Hossack, "Ultrasound despeckling for contrast enhancement," IEEE Trans. Image Process., vol. 19 , no. 7 , pp. $1847-1860,2010$

[18] O. Michailovich and A. Tannenbaum, "Blind deconvolution of medical ultrasound images: A parametric inverse filtering approach," IEEE Trans. Image Process., vol. 16, no. 12, pp. 3005-3019, 2007.

[19] Z. Chen, A. Basarab, and D. Kouamé, "Compressive deconvolution in medical ultrasound imaging," IEEE Trans. Med. Imaging, vol. 35, no. 3, pp. 728-737, 2016.

[20] H. Liebgott, R. Prost, and D. Friboulet, "Pre-beamformed RF signal reconstruction in medical ultrasound using compressive sensing," Ultrasonics, vol. 53, no. 2, pp. 525-533, 2013.

[21] O. Lorintiu, H. Liebgott, M. Alessandrini, O. Bernard, and D. Friboulet, "Compressed sensing reconstruction of 3D ultrasound data using dictionary learning and line-wise subsampling," IEEE Trans. Med. Imaging, vol. 34, no. 12, pp. 2467-2477, 2015.

[22] C. Quinsac, A. Basarab, and D. Kouamé, "Frequency domain compressive sampling for ultrasound imaging," Adv. Acoust. Vib., vol. 2012, 2012.

[23] A. Besson, R. E. Carrillo, D. Perdios, E. F. Bezzam, M. Arditi, Y. Wiaux, and J.-P. Thiran, "Morphological component analysis for sparse regularization in plane wave imaging," in IEEE Int. Ultrason. Symp., 2016, pp. 1-4.

[24] T. Szasz, A. Basarab, and D. Kouame, " $\ell_{1}$-norm regularized beamforming in ultrasound imaging," in IEEE Int. Ultrason. Symp., 2016, pp. 1-3.

[25] E. Ozkan and O. Goksel, "Inverse problem of ultrasound beamforming with sparsity in time and frequency domain," in IEEE Int. Ultrason. Symp., 2016, pp. 1-3.

[26] E. Bodnariuc, M. Schiffner, S. Petra, and C. Schnorr, "Plane wave acoustic superposition for fast ultrasound imaging," in IEEE Int. Ultrason. Symp., 2016, pp. 1-4.

[27] O. Michailovich and D. Adam, "A novel approach to the 2-D blind deconvolution problem in medical ultrasound," IEEE Trans. Med. Imaging, vol. 24, no. 1, pp. 86-104, 2005.

[28] A. R. Selfridge, G. S. Kino, and B. T. Khuri-Yakub, "A theory for the radiation pattern of a narrow-strip acoustic transducer," Appl. Phys. Lett., vol. 37, no. 1, p. 35, 1980.

[29] G. E. Tupholme, "Generation of acoustic pulses by baffled plane pistons," Mathematika, vol. 16, no. 02, p. 209, 1969.

[30] P. R. Stepanishen, "The time-dependent force and radiation impedance on a piston in a rigid infinite planar baffle," J. Acoust. Soc. Am. vol. 49, no. 1A, pp. 76-76, 1971.

[31] J. A. Jensen and N. B. Svendsen, "Calculation of pressure fields from arbitrarily shaped, apodized, and excited ultrasound transducers," IEEE Trans. Ultrason. Ferroelectr. Freq. Control, vol. 39, no. 2, pp. 262-267, 1992.

[32] A. Besson, R. E. Carrillo, D. Perdios, M. Arditi, O. Bernard, Y. Wiaux, and J.-P. Thiran, "A compressed beamforming framework for ultrafast ultrasound imaging," in IEEE Int. Ultrason. Symp., 2016.

[33] T. Szasz, A. Basarab, and D. Kouamé, "Beamforming through regularized inverse problems in ultrasound medical imaging," IEEE Trans. Ultrason. Ferroelectr. Freq. Control, vol. 63, no. 12, pp. 2031-2044, 2016.

[34] P. L. Combettes, L. Condat, J.-C. Pesquet, and B. C. Vu, "A forward-backward view of some primal-dual optimization methods in image recovery," in IEEE Int. Conf. Image Process., 2014, pp. $4141-4145$

[35] D. L. Donoho, "Compressed sensing," IEEE Trans. Inf. Theory, vol. 52, no. 4, pp. 1289-1306, 2006.

[36] E. Candès, "Compressive sampling," in Proc. Int. Congr. Math., Madrid, 2006, pp. 1433-1452.

[37] M. Fornasier and H. Rauhut, "Compressive sensing," in Handb. Math. Methods Imaging. New York, NY: Springer New York, 2011, pp. 187-228.

[38] L. Welch, "Lower bounds on the maximum cross correlation of signals (Corresp.)," IEEE Trans. Inf. Theory, vol. 20, no. 3, pp. 397-399, 1974.

[39] R. E. Carrillo, J. D. McEwen, D. Van De Ville, J.-P. Thiran, and Y. Wiaux, "Sparsity averaging for compressive imaging," IEEE Signal Process. Lett., vol. 20, no. 6, pp. 591-594, 2013. 
[40] H. Liebgott, A. Rodriguez-Molares, F. Cervenansky, J. Jensen, and O. Bernard, "Plane-wave imaging challenge in medical ultrasound," in IEEE Int. Ultrason. Symp., 2016, pp. 1-4.

[41] G. Montaldo, M. Tanter, J. Bercoff, N. Benech, and M. Fink, "Coherent plane-wave compounding for very high frame rate ultrasonography and transient elastography," IEEE Trans. Ultrason. Ferroelectr. Freq. Control, vol. 56, no. 3, pp. 489-506, 2009.

[42] C. Papadacci, M. Pernot, M. Couade, M. Fink, and M. Tanter, "Highcontrast ultrafast imaging of the heart," IEEE Trans. Ultrason. Ferroelectr. Freq. Control, vol. 61, no. 2, pp. 288-301, 2014.

[43] M. C. Van Wijk and J. M. Thijssen, "Performance testing of medical ultrasound equipment: Fundamental vs. harmonic mode," Ultrasonics, vol. 40, no. 1-8, pp. 585-591, 2002.

[44] F. Varray, M. Azizian Kalkhoran, and D. Vray, "Adaptive minimum variance coupled with sign and phase coherence factors in IQ domain for plane wave beamforming," in IEEE Int. Ultrason. Symp., 2016, pp. $1-4$.

[45] A. M. Deylami, J. A. Jensen, and B. M. Asl, "An improved minimum variance beamforming applied to plane-wave imaging in medical ultrasound," in IEEE Int. Ultrason. Symp., 2016, pp. 1-4.

[46] G. David, J.-L. Robert, B. Zhang, and A. F. Laine, "On de-cluttering and de-noising ultrasound images with time-domain compressive beamforming," in IEEE Int. Ultrason. Symp., 2016, pp. 1-4.

[47] M. Elad, "Optimized projections for compressed sensing," IEEE Trans. Signal Process., vol. 55, no. 12, pp. 5695-5702, 2007.

[48] J. Franco, G. Bernabé, J. Fernández, and M. E. Acacio, "A parallel implementation of the 2D wavelet transform using CUDA," in 2009 17th Euromicro Int. Conf. Parallel, Distrib. Network-based Process., 2009, pp. 111-118.

[49] U. S. Kamilov and H. Mansour, "Learning optimal nonlinearities for iterative thresholding algorithms," IEEE Signal Process. Lett., vol. 23, no. 5, pp. 747-751, 2016.

[50] M. A. Davenport, A. K. Massimino, D. Needell, and T. Woolf, "Constrained adaptive sensing," IEEE Trans. Signal Process., vol. 64, no. 20, pp. 5437-5449, 2016.

[51] A. Liutkus, D. Martina, S. Popoff, G. Chardon, O. Katz, G. Lerosey, S. Gigan, L. Daudet, and I. Carron, "Imaging with nature: Compressive imaging using a multiply scattering medium," Sci. Rep., vol. 4, 2014.

[52] K. Gregor and Y. LeCun, "Learning fast approximations of sparse coding," in Proceedings of the 27th International Conference on Machine Learning (ICML-10), 2010, pp. 399-406.

[53] P. Thevenaz, T. Blu, and M. Unser, "Interpolation revisited," IEEE Trans. Med. Imaging, vol. 19, no. 7, pp. 739-758, 2000. 